\title{
EL EFECTO DE LOS IMPUESTOS SOBRE EL COSTO DE CAPITAL: ESTUDIO DEL CASO ALMACENES ÉXITO S.A. (2006 -2010)*
}

\author{
EDISON JAIR DUQUE OLIVA** \& MARIA DEL PILAR BÁEZ ROA**** \\ UNIVERSIDAD NACIONAL DE COLOMBIA
}

Recibido/ Received/ Recebido: 27/02/2012 - Aceptado/ Accepted / Aprovado: 01/10/2012

\section{Resumen}

\begin{abstract}
El presente trabajo pretende, mediante el estudio de caso de la empresa Almacenes Éxito S.A. (período 2006-2010), determinar el efecto que tienen los impuestos en el costo de capital, tomando como referencia las principales proposiciones de Modigliani \& Miller (1958, 1963). Los resultados obtenidos muestran, que para el período de estudio, el efecto de los impuestos corporativos no genera una disminución del costo de capital la empresa. De tal manera, para esta entidad la contratación de deuda no tiene un beneficio tributario. Concluyendo, que para la organización, el incentivo de buscar financiación con deuda, es una decisión de su estrategia de inversión. Este documento hace un aporte a los trabajos que analizan los impuestos desde una perspectiva económica relacionando las perspectivas internas de las organizaciones.
\end{abstract}

Palabras clave: Costo de capital, Impuestos corporativos, Beneficio tributario, Deuda.

\section{EFFECT OF TAXES ON CAPITAL COST: CASE STUDY OF ALMACENES ÉXITO S.A. (2006 -2010)}

\begin{abstract}
The purpose of this paper is to determine the effect that taxes have on capital cost through the case study of Almacenes Éxito S.A. (period 2006-2010), considering as reference of study the main premisesof Modigliani \& Miller $(1958,1963)$. The main findings show that the effect of taxes does not decreases the capital cost of a company in the period of study. Therefore, debt contracting does not have a tributary benefit for this company. This allows concluding that for the corporation, the incentive to access to financing through debt is a decision of its investment strategy. This paper contributes to other works that analyze taxes since an economic approach relating corporation's internal perspectives.
\end{abstract}

Keywords: Capital cost, Corporative taxes, Tributary benefit, Debt.

\footnotetext{
* El presente artículo es producto del trabajo de grado titulado "El efecto de los impuestos sobre el costo de capital: estudio de un caso en Colombia -Almacenes Éxito S.A. Periodo 2006 -2010", Universidad Nacional de Colombia, realizado en Noviembre de 2011.

** PhD en Marketing. Universitat de Valencia Estudi General, Valencia, MBA concentración en mercadeo Universidad de los Andes Bogotá, Master in Economics and Management MSc. (official language: English), Universitat Pompeu Fabra, Barcelona, Administrador de Empresas Universidad Nacional de Colombia, Bogotá. Profesor Asociado. Facultad de Ciencias Económicas Universidad Nacional de Colombia. Grupo GRIEGO. Correo electrónico: jair ejduqueo@unal.edu.co

*** $\quad$ Master en Administración, Universidad Nacional de Colombia. Especialista en Ingeniería del Software Universidad INCCA de Colombia Bogotá, Especialista en Finanzas Universidad Pedagógica y Tecnológica de Colombia Sogamoso. Profesora Universidad Pedagógica y Tecnológica de Colombia. Grupo GRINDEP. Correo electrónico: mariabaez11@gmail.com
} 


\title{
O EFEITO DOS IMPOSTOS SOBRE O CUSTO DE CAPITAL: ESTUDO DE CASO DOS ARMAZÉNS ÉXITO S.A. (2006 -2010)
}

\section{Resumo}

\begin{abstract}
Através do estudo de caso da empresa Armazenes Éxito S.A. (período 2006-2010), o presente trabalho pretende determinar os efeitos que têm os impostos no custo de capital, tomando como referência as principais proposições de Modigliani e Miller $(1958,1963)$. Os resultados obtidos mostram que para o período de estudo, o efeito dos impostos corporativos no custo de capital da empresa não tem um significativo benefício tributário ao contratar com dívida. Conclui-se que para a organização o incentivo de procurar financiamento com dívida é uma decisão de sua estratégia de investimento. Este documento contribui com os trabalhos que analisam os impostos desde uma perspectiva econômica relacionando as perspectivas internas das organizações.
\end{abstract}

Palavras chave: Custo de capital, Impostos corporativos, Benefício tributário, Dívida.

Duque, E. \& Báez, M. (2012) El efecto de los impuestos sobre el costo de capital: estudio del caso Almacenes Éxito (2006 -2010). En: Revista de la Facultad de Ciencias Económicas de la Universidad Militar Nueva Granada. rev.fac.cienc.econ, XX (2).

JEL: G30, G32.

\section{Introducción}

El sistema tributario colombiano es complejo porque cuenta con una diversidad de tratamientos especiales entre los que se encuentran exenciones, descuentos, deducciones, tarifas diferenciales y continuas reformas. Dada esta diversidad y desde la perspectiva de la gestión financiera, algunas reformas pueden aportar al incremento de los flujos de caja de las empresas o a disminuir su costo de capital, mediante incentivos tributarios que incrementen su valor ${ }^{1}$. Dentro de las reformas que han afectado negativamente a los flujos de caja y al costo de capital de las empresas tenemos el impuesto a las transacciones financieras. Este en su momento afecto el crecimiento de los créditos ofrecidos por el sistema financiero ya que las dos terceras partes la pagaban los usuarios; la sobretasa del $10 \%$ en el impuesto de renta (2002-2006) lo que hizo que éste ascendiera al 38,5\%. (Clavijo, 2005, 2).

Por otro lado, las reformas tributarias también han afectado los flujos de caja del gobierno, cuando al colocar altas cargas impositivas o buscando bajarlas a través de múltiples exenciones, estas pueden en últimas aumentar el déficit de la nación por lo cual debe recurrir a inversiones de deuda pública para suplir su déficit. Como se puede observar el tema tributario reviste una gran importancia en la gestión financiera tanto de las organizaciones públicas como privadas.

El estudio del costo de capital, como costo de las fuentes de financiación que la empresa necesita para su operación, es fundamental porque de él dependen las decisiones de inversión en capital físico productivo. De igual manera, el costo de capital permite establecer si un proyecto de inversión genera valor a la firma o si lo destruye, pues este es la tasa mínima requerida sobre un proyecto de inversión.

Por otro lado, permite determinar cómo será financiado el proyecto en términos de deuda y/o capital y sus implicaciones (las fuentes de financiación tienen diferentes tipos de riesgo así: los accionistas comprometen su capital, y en caso de una quiebra son los últimos en la lista en recuperar su capital, mientras que los acreedores tienen una menor probabilidad

1 En la teoría financiera el valor de una compañía corresponde entre otros aspectos a la estimación del valor presente de sus flujos de caja descontados a una tasa de descuento que podría ser el costo de capital. 
de perder su dinero). Finalmente, el costo de capital de la firma es una tasa de referencia que mide el riesgo de mercado de las posibles oportunidades para financiar las inversiones de las empresas y una herramienta para asignar los recursos de una manera más eficiente (costo de oportunidad de los financiadores).

Como se puede observar, el costo de capital es un concepto importante en la gestión financiera de las empresas. Por lo cual el presente estudio ha seleccionado uno de sus determinantes, como son los impuestos. Siendo, la pregunta de investigación: ¿cuál es el efecto de los impuestos corporativos en el costo del capital de Almacenes Éxito S.A.? Esta inquietud ha sido tratada por la teoría y existen modelos teóricos a través de los cuales se ha analizado el fenómeno. Dada su complejidad sería difícil hacer un análisis macro y por tanto, se establece una compañía representativa para este estudio. La empresa Almacenes Éxito S.A. es la más grande y con mayor participación en el mercado del sector comercio al por menor en Colombia, participando con el $12 \%$ del PIB colombiano, y generando el $25,6 \%$ del empleo total ${ }^{2}$, indicando que tiene una participación importante en la economía del país.

El objetivo del presente estudio es determinar el efecto de los impuestos corporativos en el costo de capital en el caso Almacenes Éxito S.A. período 2006 - 2010. Para el desarrollo de este estudio fue necesario conocer un poco más a profundidad algunos aspectos del caso Almacenes Éxito S.A. a saber: un análisis de sus estados financieros que permita visualizar la generalidad del caso; caracterizar la metodología del modelo CAPM (Capital Assets Pricing Model) ajustado y el modelo APT (Arbitrage Pricing Theory) utilizada para el cálculo del costo del patrimonio, establecer la metodología a aplicar en la determinación del efecto de los impuestos en el costo de capital; calcular el costo de capital a partir de las metodologías CAPM ajustado y APT utilizadas en el cálculo del costo del patrimonio; y finalmente contestar a la pregunta ¿̇cuál es el efecto de los impues- tos corporativos en el costo del capital de Almacenes Éxito S.A.?

\section{Conceptos fundamentales}

\subsection{Costo de capital}

Cuando se invierte en una firma es necesario recurrir a diferentes fuentes de financiación las cuales tienen un costo por su uso. La procedencia de dicha fuentes, son principalmente la deuda y los recursos propios, a este costo se denomina costo de capital de la firma, que se calcula ponderando el costo de cada tipo específico de fuente con su proporción en el valor total de la firma. García (2003, 247) lo define como "la rentabilidad mínima que deben producir los activos de una empresa".

El cálculo del costo promedio ponderado de capital se efectúa según la ecuación (1):

$C k=\left(\frac{E}{V}\right) K e+\left(\frac{D}{V}\right) K d(1-t)$

Donde:

$C k=$ Costo de capital

$E=$ Valor de los recursos propios o patrimonio

$D=$ Valor de la deuda

$V=E+D$ valor total de la firma

$\mathrm{Ke}=$ Costo de los recursos propios

$K d=$ Costo de la deuda

$t=$ Tasa impositiva

\subsubsection{Costo de la deuda}

El costo de la deuda es el rendimiento que exigen los acreedores por el uso de sus recursos que está representado en los intereses y el pago del principal. Gitman (2007) determina que el costo de la deuda es el costo actual después de impuestos de obtener fondos a largo plazo mediante endeudamiento. El costo de la deuda se calcula dividiendo lo que se paga (intereses) sobre los recursos utilizados. Los intereses de los acreedores financieros se deducen del

2 Informes económicos Interbolsa. Extraídos de: http://www.interbolsa.com/ 
ingreso gravable de la firma generando una menor base para el pago de los impuestos y por lo tanto unos menores impuestos. Es así que el costo de la deuda se determina de la siguiente forma:

$K d_{t}=K d(1-t)$

Donde:

$K d=$ Costo de la deuda

$K d_{t}=$ Costo de la deuda después de impuestos

$t=$ Tasa impositiva

El riesgo de la deuda es menor al riesgo del patrimonio debido a que la deuda tiene prioridad sobre los pagos de utilidades o dividendos de los accionistas, además se exigen garantías y codeudores en caso de que la firma no pueda pagar, disminuyéndose así el riesgo de no pago. Por lo tanto, el costo de la deuda debe ser menor al costo del patrimonio.

\subsubsection{Costo de los recursos propios o accionario}

El costo de los recursos propios es el rendimiento requerido por los accionistas o inversionistas por haber invertido en la firma, los cuales pueden ser recibidos en forma de dividendos y ganancias de capital. Los tenedores de las acciones son los propietarios de la firma. El costo de los recursos propios tiene una remuneración residual es decir, se paga después de pagar todas las obligaciones de la firma, en caso de quedar un remanente. En caso de quiebra son los últimos en recibir su dinero. Es por esto que los accionistas corren un mayor riesgo que los acreedores, por lo que su costo debe ser mayor para compensar ese mayor riesgo.

El cálculo del costo de los recursos propios o accionario es uno de los más complejos en el cálculo del costo de capital. El modelo más utilizado para su cálculo es la metodología de valuación de activos de capital, CAPM, que depende de la tasa libre de riesgo, la prima del mercado y el riesgo no diversificable de la empresa medido por el coeficiente beta. En este modelo la rentabilidad de una acción está relacionada en forma lineal:

$$
K_{e}=R_{f}+\beta\left(R_{m}-R_{f}\right)
$$

Donde:

$K e=$ Rendimiento esperado por el inversionista o accionista

$R f=$ Tasa libre de riesgo

$R m-R f=$ Prima de riesgo de mercado

$\beta=$ Beta de la firma

La tasa libre de riesgo corresponde a los bonos del tesoro nacional TES, porque se considera la empresa como el gobierno de la nación. La prima de riesgo del mercado es la rentabilidad adicional que un inversionista obtiene por invertir en acciones y no en TES. Beta es "una medida de riesgo que asocia la volatilidad de la rentabilidad de una acción con la volatilidad de la rentabilidad del mercado" (García, 2003, 254).

Su aplicación se refiere a mercados desarrollados, siendo esta una limitante en países en vía de desarrollo como Colombia ya que el grupo de empresas que transan en el mercado público es muy reducido, esto "implica que muy pocos sectores de la actividad económica hacen presencia en el mercado público $y$ los que hacen presencia están conformados por muy pocas empresas, con lo que las betas sectoriales o no existirían o no serían confiables con muestras poco representativas" (García, 2003, 261). Sin embargo, la alternativa que surge para aplicar el modelo CAPM es referenciar información de países con mercados bursátiles más grandes y eficientes como EE.UU.

Por lo que para calcular el costo accionario con las cifras de EE.UU, se debe apalancar el beta del sector con el endeudamiento correspondiente a la empresa que se va a analizar y se ajusta el resultado con el riesgo país para reflejar el riesgo adicional por realizarse en otro país y no en EE.UU. El resultado se

3 Formula de Hamada. 
obtendría en dólares siendo necesario aplicar la devaluación esperada de largo plazo. Para apalancar la beta del sector con el endeudamiento se utiliza la la ecuación $(4)^{3}$.

$\beta_{L}=\beta_{u}[1+(1-t) D / E]$

Donde:

$\beta_{L}=\beta$ apalancado

$\beta_{u}=\beta$ desapalancado $\mathrm{u}$ operativo

$t^{u}=$ Tasa de impuesto

$D / E=$ Relación de apalancamiento

Existe otra metodología para el cálculo del costo del patrimonio desarrollada por Ross (1976), el modelo para la valuación de activos de capital APT. Ross et al. (2003) definen que el retorno de cualquier activo riesgoso depende de una combinación lineal de varios factores los cuales aún no están determinados. Sin embargo, los factores más comunes que se mencionan en el modelo APT y que deben estar relacionados con variables macroeconómicas son los cambios en el producto interno bruto, los cambios en la inflación y los cambios en las tasas de interés.

Esta metodología dice que el rendimiento de una acción está formado por dos partes:

- El rendimiento normal o esperado: es la parte que los accionistas predicen o esperan, dependen de la información que los accionistas tengan.

- Rendimiento incierto o riesgoso: proviene de la información que no tienen los accionistas.

Por consiguiente, los rendimientos de una acción quedarían expresados en términos de:

$R=X+U$

Donde:

$X=$ Parte esperada del rendimiento

$U=$ Parte no esperada del rendimiento

Además, divide la parte de factor sorpresa en riesgo sistemático y riesgo operativo así:
$R=X+m+e$

Donde:

$m=$ Riesgo sistemático

$e=$ Riesgo no sistemático o propio de la empresa

Ahora, el $m$ puede estar conformado por varios riesgos sistemático que influyen en el rendimiento de la acción, los cuales pueden ser PIB, tasa de interés e inflación por lo que cada una tendrá una beta asociada a ella. Así que, reescribiendo $R$ se tiene la ecuación (7).

$R=X+\beta F_{1}+\beta F_{2}+\beta F_{3}+e$

Pueden existir diferentes versiones de la especificación del modelo, dependiendo en últimas, de cuál de ellas arroja los mejores resultados empíricos, en términos de su capacidad para reproducir el comportamiento de las tasas de retorno de los activos.

Los coeficientes betas nos indican la respuesta del rendimiento de una acción ante un riesgo sistemático, es decir que tan sensible es el rendimiento de una acción a los cambios no anticipados de la inflación. Los factores determinan las novedades o sorpresas ocurridas.

Uno de los métodos para determinar los factores comunes es las variables macroeconómicas en las que el investigador basado en su propio juicio escoge factores, para luego estimar los coeficientes de sensitividad y verificar, si ellos explican el corte transversal de los retornos (Chan, Chen \& Hsieh, 1985; Chen, et al., 2003; y Chen \& Jordan (1993); citados por Fuentes, Gregoire \& Zurita, 2005). El método de las variables macroeconómicas tiene la ventaja de permitir la interpretación económica de los factores y los premios por riesgos factoriales.

\section{Marco teórico}

\subsection{El efecto de los impuestos en el costo de capital}

Para poder obtener la expresión del costo de capital ajustado por impuestos, es necesario conocer los es- 
tudios pioneros de Modigliani \& Miller (1958, 1963) respecto a los factores que pueden afectar el valor de la firma. A continuación se hará una breve explicación de sus principales proposiciones.

\subsubsection{Proposición I: Modigliani E Miller (1958) -sin impuestos corporativos-}

Modigliani \& Miller (1958) en su primera proposición determinan que el valor de mercado de la firma depende de sus rendimientos esperados descontados a una tasa $k$ apropiada para su clase sin tener ninguna influencia cuáles son sus fuentes de financiación. Lo que nos indica que el valor de la empresa está representado por las utilidades antes de interés e impuestos (UAII) descontadas al costo de capital (Ck) y que este costo de capital permanece constante ante cualquier cambio en el nivel de deuda. Lo que se podría expresar en la ecuación (8).

$$
V=\frac{U A I I}{C k}
$$

Para demostrar la anterior afirmación, aplican el concepto de arbitraje a un ejemplo de dos empresas del mismo sector, con el mismo riesgo y con utilidades operacionales esperadas iguales, pero con una estructura de capital diferente que tienen el mismo valor. Por lo tanto, si el valor de estas dos empresas fueran diferentes el inversionista de la empresa sobrevalorada podría comprar una participación de la empresa subvalorada a un costo más bajo y obtendría el mismo rendimiento. De esta forma los inversionistas aprovecharían las oportunidades de arbitraje hasta el punto que harían que las participaciones de la empresa subvalorada subieran y las participaciones de la empresa sobrevalorada bajaran hasta volver al equilibrio.

\subsubsection{Proposición II: Modigliani \& Miller (1958) -sin impuestos corporativos-}

La proposición II relaciona directamente el rendimiento esperado sobre el capital social de una firma apalancada con el nivel de endeudamiento mediante la ecuación (9).

$k_{s}=k_{o}+\left(k_{o}-k_{d}\right) \frac{D}{E}$
Donde:

$D=$ Valor de la deuda

$E=$ Valor de las acciones o del capital social

$k_{s}=$ Costo del capital social de una empresa apalancada

$k_{\mathrm{o}}=$ Costo de una empresa totalmente financiada con recursos propios.

$K_{d}=$ Costo de la deuda

Como podemos observar en la ecuación (9), el valor del costo del capital social está directamente relacionado con la proporción de deuda, es decir, a medida que incrementamos la deuda el costo del capital social aumenta debido al mayor riesgo en que se incurre, anulando las economías obtenidas por el mayor valor de deuda lo que hace que el costo del capital se mantenga constante. Llegando a concluirse que la estructura de capital de la firma no afecta el valor de la empresa.

\subsubsection{Proposición I: Modigliani \& Miller (1963) -con impuestos corporativos-}

En esta tesis los autores consideran el efecto de los impuestos en la estructura de capital. Con ésta corrigieron su Proposición I, reconociendo las grandes ventajas tributarias en el valor de la empresa así:

- En una empresa no apalancada el flujo de efectivo (UAII) después de impuestos es: ).

- En una empresa apalancada el flujo de efectivo después de impuestos es:

El valor de una empresa no apalancada, es decir, financiada totalmente con recursos propios es el valor presente de sus flujos de efectivo descontada al costo de su capital social (ecuación 10).

$$
V_{u}=\frac{U A I I(1-T c)}{k_{o}}
$$

Donde:

$V_{u} \quad=$ Valor presente de una empresa

UAII = Utilidades antes de intereses e impuestos

$T c=$ Tasa fiscal corporativa 
$k_{\circ} \quad=$ Costo de capital de una empresa totalmente financiada con recursos propios.

$k_{d} \quad=$ Costo de la deuda

$D^{d}=$ Deuda

Para determinar el valor de una empresa apalancada $\left(V_{L}\right)$, es necesario adicionar el valor presente de la protección fiscal proveniente de la deuda (ecuación 11).

$$
V_{L}=\frac{U A I I(1-T c)}{k_{o}}+\frac{T_{c} k_{d} D}{k_{d}}
$$

Reemplazando (10) en (11) se tiene que:

$$
V_{L}=V_{U}+T_{C} B
$$

Como podemos observar el mayor valor de una empresa apalancada se encontraría cuando está financiada totalmente con deuda. Sin embargo en la vida real las personas no se pueden endeudar en un cien por ciento, ya que esta situación daría señales negativas al mercado y generaría unos costos de quiebra. Teniendo en cuenta lo anterior, se replantea las Proposiciones I y II de Modigliani \& Miller (1958, 1963) con impuestos corporativos (ecuación 13).

$$
\frac{U A I I_{V L}}{V_{L}}=K_{O}-T_{C}\left(K_{O}-K_{d}\right) \frac{D}{V_{L}}
$$

Además de la Proposición II se replantea así:

$$
k_{s}=k_{o}+\frac{D}{E}\left(1-T_{c}\right)\left(k_{o}-k_{d}\right)
$$

Donde, $k_{s}$ es el costo del capital social de una empresa apalancada. Para ver en más detalles acerca de la derivación matemática de las fórmulas ver Anexo 1.

Ahora, al costo de la deuda se le aplica el efecto del impuesto multiplicado por $\left(1-\mathrm{T}_{c}\right)$ porque los intereses son deducibles de impuestos, quedando el costo de capital con impuestos corporativos así:

$$
C k=\frac{B}{V_{L}} k_{d}\left(1-T_{C}\right)+\frac{E}{V_{L}} k_{S}
$$

\subsection{Tasas impositivas}

El sistema fiscal colombiano se encuentra regulado por el Decreto No. 624 de 1989, denominado "Estatuto Tributario". El sistema tributario comprende impuestos de carácter nacional, departamental y municipal. El impuesto sobre la renta y sus complementarios es un tributo de carácter nacional y es definida legalmente por el gobierno. La base sometida es la renta gravable, que se obtiene de sumar todos los ingresos ordinarios y extraordinarios susceptibles de producir un incremento neto del patrimonio y que no estén expresamente exceptuados en la ley. De esta suma se restan las devoluciones, rebajas y descuentos, así como los costos y deducciones imputables a tales ingresos.

Con la Ley 1111 expedida el 27 de diciembre de 2006, se encuentran las disposiciones por las cuales se reduce la tarifa del impuesto sobre la renta que aplican a partir del año 2007, se disminuyen gradualmente a partir del año gravable 2007, de este modo: año gravable 2007 es 34\%, y año gravables 2008 y subsiguientes es $33 \%$.

Para medir la participación estatal en las utilidades de la empresa utilizamos la tasa efectiva de tributación la cual puede variar periódicamente dependiendo de factores tales como: gastos no deducibles, uso de beneficios fiscales, uso de partidas compensatorias, etc.

La tasa marginal del impuesto corresponde al cambio en el impuesto total dividido entre el cambio en el ingreso.

Las fórmulas fueron tomadas con base al estudio de Herrera \& Mora (1998), modificando la utilidad bruta por la utilidad antes de impuestos necesario para nuestro estudio (ecuaciones 16 y 17).

$$
\begin{aligned}
& t_{e}=\frac{(\text { Impuestos Pagados }+ \text { Cambio en los Impuestos por pagar (de signo positivo)) }}{\text { Utilidad antes de impuestos }} \\
& t_{m}=\frac{\text { Cambio en los impuestos pagados y en los impuestos por pagar }}{\text { Cambio en la utilidad antes de impuestos }}
\end{aligned}
$$




\section{Metodología}

Este estudio es empírico-analítico, donde existe una realidad fáctica, externa y objetiva que se observa por parte del investigador (caso Almacenes Éxito S.A. ${ }^{4}$ ) y donde hay independencia del investigador. La concepción de la realidad en un estudio de este tipo se hace de manera a-histórica y fragmentada y para este caso específico se analiza el periodo 2006 - 2010. El método de investigación es el estudio de caso, es decir, el estudio de una sola unidad de análisis en su entorno práctico, permitiendo la posibilidad de comprender la naturaleza de la contabilidad y las finanzas en la práctica (Ryan, Scapen \& Theobald, 2004).

El tipo de estudio utilizado es útil para comprender la situación actual de Almacenes Éxito S.A., en cuanto a sus estrategias y planteamientos financieros, y para determinar el efecto de los impuestos en el costo de capital, bajo la siguiente pregunta guía, ¿̇cuál es el efecto de los impuestos corporativos en el costo de capital del caso Almacenes Éxito S.A. período 2006 - 2010?

Se parte entonces de entender el comportamiento de la empresa, en términos tanto de sus estrategias como de sus planteamientos y situación financiera. Esto se hizo mediante un análisis horizontal que permitió observar las principales variaciones de los estados financieros dentro de un período de tiempo determinado. También se observó el impacto de las estrategias desarrolladas en la rentabilidad de la empresa y su desarrollo en el periodo de estudio. De esta forma podemos tener una primera aproximación de la evolución de los impuestos en el período de estudio y sus causas. En segundo lugar se define el valor de los elementos que componen el costo de capital para Almacenes Éxito S.A como son el costo de capital accionario utilizando la metodología CAPM y APT, el costo de la deuda y el costo de los bonos. En tercer lugar, se calcularon las tasas efectivas y marginales de impuestos corporativos para la empresa observando las principales variables que las afectan. Para luego aplicarlas al costo de capital y compararlas con el costo de capital sin impuestos y analizar los cambios.

La información necesaria para el estudio (informes financieros, e investigaciones económicas) se obtuvo del sistema construido por la Superintendencia de Sociedades - SIREM (ver Anexo 2).

\subsection{Costo de capital}

Para calcular el costo de capital, es necesario conocer los costos de cada una de las fuentes de financiación de Almacenes Éxito S.A.

\subsubsection{Cálculo del costo accionario utilizando el modelo CAPM ajustado}

A continuación se detallará las fuentes de información utilizadas para el cálculo del costo accionario:

- Para el cálculo del coeficiente de riesgo beta, se tomó información histórica semanal de los precios de las acciones de Almacenes Éxito S.A. y del índice general de la bolsa de valores de Colombia (IGBC) como referente del mercado para el período de estudio obtenida de las bases de Thomson Reuters a través del aplicativo EIKON, provisto por la Unidad de Análisis del Mercado Financiero de la Facultad. Dichas series se convirtieron a rendimientos continuos $\operatorname{con}\left(\operatorname{Ln}\left(\left(P_{i+1}\right) / P_{i}\right)\left(\right.\right.$ Ln (indice $\left.{ }_{i+1}\right) /\left(\right.$ indice $\left._{i}\right)$ - para luego hacer una regresión lineal (del tipo $y=m x$ $+b$ ). Debido a que el beta del año 2006 no resultó significativo y el del año 2007 se obtuvo un $R^{2}$ del $11 \%$ fue necesario para estos años tomar las betas perteneciente al sector de Retail Store del profesor Damodaran ${ }^{5}$ y apalancarlas con la deuda de la empresa.

- La prima de riesgo se calcula como los promedios históricos de la rentabilidad por invertir en acciones y la rentabilidad de los bonos del tesoro. De los años 1928-2010 equivalente a 6,03\% con desviación estándar de $2,38 \%$ siendo esta

4 Estableció como estrategia de crecimiento mejorar su posición financiera mediante un programa de disminución de deuda y de esta forma mejorar sus indicadores, abriendo cupo para posibles nuevas emisiones de bonos o tomas de créditos para sostener los niveles de expansión.

5 Extraídos de: http://pages.stern.nyu.edu/ adamodar/New_Home_Page/data.html 
la menor desviación de las diferentes primas presentadas y se aplicó la devaluación.

- La rentabilidad de los bonos del tesoro de Colombia con vencimiento a 10 años.

- La devaluación se tomó de las investigaciones económicas de la sociedad comisionista Interbolsa.

\subsubsection{Cálculo del costo accionario utilizando el modelo APT}

Las fuentes sistemáticas de riesgo que hemos considerado son las variables macroeconómicas como el $\mathrm{PIB}$, la Inflación y la tasa de interés. En este modelo el rendimiento de una acción está determinado por la ecuación (18).

$K e=R f+B_{P I B}(P I B)+B_{I F}(I F)+B_{t i}(T I)+e$

Donde:

$R f=$ Rendimiento esperado o tasa libre de riesgo

$B_{P I B}=$ Beta del PIB

$B_{I F}=$ Beta inflacionaria

$B_{t i}=$ Beta de la tasa de interés

$e \quad=$ Riesgo no sistemático propio de la compañía

$P I B=$ Cambios en el PIB

IF = Cambios en la inflación

TI = Cambios en la tasa de interés

Estimando los coeficientes de riesgo por medio de mínimos cuadrados se tiene ecuación (19).

$K e=B_{P I B}(P I B)+B_{I F}(I F)+B_{t i}(T I)+e$

Las fuentes de la información recolectada fueron:

- Precios diarios de las acciones de Almacenes Éxito S.A. durante el período $2006-2010^{6}$.

- PIB trimestral de Colombia durante el período $2006-2010^{7}$.
- Inflación del trimestre durante el período 2006 $2010^{8}$.

- Tasas de interés de los certificados de depósito a término a 90 días DTF, promedios mensuales, durante el período 2006 - $2010^{9}$.

Se llevo toda la información en el mismo período trimestral, y se determinó el precio de la acción por trimestre tomando el promedio de los precios durante el trimestre correspondiente, lo mismo para la tasa de interés.

Por otro lado, la estimación de las primas de riesgo APT por medio de mínimos cuadrados, se hace mediante ecuación (20).

$K e=P I B\left(B_{P I B}\right)+I F\left(B_{I F} T I\right)+\left(B_{t i}\right)+e$

La definición de datos para tal efecto fue:

- Cambios en los precios trimestrales de las acciones: $\frac{\mathrm{P}_{\mathrm{t}-\mathrm{P}_{\mathrm{t}-1}}}{\mathrm{P}_{\mathrm{t}-1}}$ durante el período $2006-2010^{10}$.

- Cambio en el PIB trimestral: $\frac{\mathrm{PIB}_{\mathrm{t}-\mathrm{PIB}}}{\mathrm{PIB}_{\mathrm{t}-1}}$ durante el período 2006 - 2010.

- Cambio en la inflación trimestral: $\frac{\mathrm{IF}_{\mathrm{t}-\mathrm{IF}} \mathrm{IF}_{\mathrm{t}-1}}{\mathrm{I}_{\mathrm{t}}} \mathrm{du}$ rante el período 2006 - 2010.

- Cambios en el DTF trimestrales: $\frac{\mathrm{IF}_{\mathrm{t}-\mathrm{IF}}}{\mathrm{IF}_{\mathrm{t}-1}}$ durante el período 2006 - 2010.

- El valor esperado del costo accionario está dado por:

$$
E(K e)=R_{f}+\beta(\mathrm{Rm}-\mathrm{Rf})+\widehat{B}_{P I B} \widehat{P I B}+\widehat{B}_{I F} \widehat{I F}+\widehat{B}_{T I} \widehat{T I}
$$

\subsubsection{Costo de la deuda}

El costo de la deuda se tomó de las tasas de colocación de créditos comerciales del Banco de la Repú-

\footnotetext{
Extraídos de: https://www.grupoaval.com/portal/page?_pageid=33,115460184\&_dad=portal\&_schema=PORTAL Extraído de: http://www.dane.gov.co/

Extraída de: http://www.dane.gov.co/

Extraídas de: http://www.banrep.gov.co/

Extraídos de: https://www.grupoaval.com/portal/page?_pageid=33,115460184\&_dad=portal\&_schema=PORTAL
} 
blica categoría preferencial o corporativo, seleccionando las perteneciente al monto más cercano de las obligaciones financieras de Almacenes Éxito S.A. 2006-2010.

Esta fuente ha sido validada para estos estudios en el ámbito colombiano, por ejemplo, Salas, Gutiérrez \& Vélez (2011) estiman el valor de los ahorros de impuestos para 23 de las principales empresas que cotizan en bolsa entre el 2001 y el 2010 en Colombia.

\subsubsection{Costo de los bonos}

Para el cálculo del costo de los bonos se recogió la siguiente información de prospectos del SIMEV.

Tabla 1. ${ }^{11}$

\begin{tabular}{|c|c|}
\hline Bono A & DTF $+2,5 \%$ TA \\
\hline Bono B & IPC $+6 \%$ EA \\
\hline Bono C & $12,5 \%$ EA \\
\hline
\end{tabular}

A partir de la cual se realizó la conversión de tasas a efectivas para cada año de estudio y luego calcular un promedio entre los tres tipos de bonos como costo de la deuda en bonos.

Teniendo ya todos los costos se aplicó la fórmula del modelo CAPM (ecuación 3).

\section{Resultados}

\subsection{Análisis horizontal de rentabilidad}

En el análisis horizontal de la rentabilidad para el caso Almacenes Éxito en el período de estudio 2006 -2010, se observa el desarrollo de su estrategia de expansión con la adquisición de Carulla- Vivero y su estrategia financiera de reducción de deuda. Por otro lado, se encuentra un periodo de disminución de la rentabilidad en los años 2007-2009 (ver Tabla 2) tiempo de la fusión-, al parecer generado por mayores gastos operacionales y cierres de Almacenes por

11 Fuente: http://www.superfinanciera.gov.co/web_valores/Simev

12 Fuente: http://www.interbolsa.com/

Donde: $\mathrm{ROA}$ = rentabilidad del activo; $\mathrm{ROE}$ = rentabilidad del patrimonio. periodos de 3 a 4 meses (durante las remodelaciones). Finalizada la fusión se logra nuevamente incrementar los índices de rentabilidad y con proyecciones a incrementos mayores, esto como resultado del aprovechamiento de sinergias, mejoramiento operativo, eficiencias en el posicionamiento de sus marcas propias las cuales generan una mayor rentabilidad y la creación de otros negocios complementarios.

Tabla 2. Índice de rentabilidad ROE y ROA - Almacenes Éxito S.A. ${ }^{12}$

\begin{tabular}{|l|c|c|c|c|c|}
\hline $\begin{array}{c}\text { Índice de } \\
\text { rentabilidad }\end{array}$ & $\mathbf{2 0 0 6}$ & $\mathbf{2 0 0 7}$ & $\mathbf{2 0 0 8}$ & $\mathbf{2 0 0 9}$ & $\mathbf{2 0 1 0}$ \\
\hline ROA & $3,38 \%$ & $2,13 \%$ & $2,5 \%$ & $2,3 \%$ & $3,8 \%$ \\
\hline ROE & $5,94 \%$ & $3,87 \%$ & $4,5 \%$ & $3,5 \%$ & $5,8 \%$ \\
\hline
\end{tabular}

\subsection{Costo de capital: metodología CAPM ajustado}

En el Anexo 3 se encuentran las estimaciones de los betas para los años 2006 - 2010. Mientras en la Tabla 3 se observa el valor del costo de capital para la empresa Almacenes Éxito S.A. período 2006 - 2010.

Para el año 2006 el costo accionario fue de 20,44\% y costo de capital fue de $16,45 \%$, para el año 2007 el costo del capital accionario se incrementó en un $21,14 \%$ lo que hizo que también el costo de capital aumentará a un $16,78 \%$. Esto principalmente por un mayor valor de la tasa libre de riesgo y del costo de la deuda. Para el 2008, el costo del capital accionario y el costo del capital aumentaron principalmente por una mayor tasa libre de riesgo y mayores costo de la deuda Para el año 2009, el costo del capital accionario disminuyo a $18,89 \%$, principalmente por un menor tasa libre de riesgo y un menor beta, lo que hizo que el costo de capital también disminuyera. Finalmente, para el año 2010 el costo del capital accionario disminuyo debido a una disminución sustancial en el beta.

De otro lado, la estimación de los coeficientes de riesgo partió de las series diarias de los precios de Almacenes Éxito S.A., de las cuales se establecieron 
Tabla 3. Costo de Capital Metodología CAPM ${ }^{13}$

\begin{tabular}{|l|c|c|c|c|c|}
\hline $\begin{array}{c}\text { Variables e } \\
\text { indicadores }\end{array}$ & $\mathbf{2 0 0 6}$ & $\mathbf{2 0 0 7}$ & $\mathbf{2 0 0 8}$ & $\mathbf{2 0 0 9}$ & $\mathbf{2 0 1 0}$ \\
\hline Beta & 1,23 & 1,2 & 1,23 & 1,11 & 0,69 \\
\hline Prima de mercado & $9,21 \%$ & $9,21 \%$ & $9,21 \%$ & $8,68 \%$ & $8,68 \%$ \\
\hline Tasa libre de riesgo & $9,11 \%$ & $10,09 \%$ & $11,71 \%$ & $9,26 \%$ & $7,76 \%$ \\
\hline$D$ & 254.363 .930 & 955.290 .250 & 477.506 .539 & 283.663 .944 & 0 \\
\hline$E$ & 599.726 .244 & 1.589 .649 .107 & 1.589 .649 .107 & 2.291 .551 .525 & 2.292 .928 .719 \\
\hline$D B$ & 105.000 .000 & 105.000 .000 & 105.000 .000 & 105.000 .000 & 224.650 .000 \\
\hline$E / E+D+D B$ & $62,53 \%$ & $59,99 \%$ & $73,18 \%$ & $85,50 \%$ & $91,08 \%$ \\
\hline$D / E+D+D B$ & $26,52 \%$ & $36,05 \%$ & $21,98 \%$ & $10,58 \%$ & $0,00 \%$ \\
\hline$D B / E+D+D B$ & $10,95 \%$ & $3,96 \%$ & $4,83 \%$ & $3,92 \%$ & $8,92 \%$ \\
\hline$K s$ & $20,44 \%$ & $21,14 \%$ & $23,04 \%$ & $18,89 \%$ & $13,75 \%$ \\
\hline$K d$ & $9,41 \%$ & $10,08 \%$ & $11,30 \%$ & $11,60 \%$ & $11,60 \%$ \\
\hline$K_{\text {bonos }}$ & $10,72 \%$ & $11,75 \%$ & $13,03 \%$ & $9,81 \%$ & $9,38 \%$ \\
\hline Costo de Capital & $16,45 \%$ & $16,78 \%$ & $19,98 \%$ & $17,76 \%$ & $13,36 \%$ \\
\hline
\end{tabular}

los valores para el trimestre (90 observaciones en promedio por trimestre) y se obtiene una serie de 32 observaciones trimestrales que recogen las observaciones diarias (2900 días aproximadamente). Siendo el número de observaciones más representativas teniendo en cuenta la teoría.

Sin embargo, los resultados no cambiaron sustancialmente. Inicialmente se realizó la regresión de la variable rentabilidad de la acción del Éxito con la variable Inflación siendo significativo pero su coeficiente de determinación estaba muy cercano a cero por lo que no era muy confiable. Por lo tanto, al realizar la regresión en su conjunto ya no era significativa.

\subsection{Costo de capital: metodología APT}

La estimación de los coeficientes de riesgo partió de las series diarias de los precios de las acciones de
Almacenes Éxito, de las cuales se establecieron los valores para el trimestre.

Utilizando los datos de la Tabla 4 se realizó la prueba de significancia y el intervalo de confianza para los betas e interpretar $\left(t_{\propto / 2}\right)$, encontrándose que el único beta significativo es el correspondiente a la variable del PIB. En cuanto a las otras variables, éstas no fueron significativas.

Tabla 4. Estimación coeficientes de riesgo ${ }^{14}$

\begin{tabular}{|l|c|}
\hline \multicolumn{2}{|c|}{ Estadísticas de la regresión } \\
\hline Coeficiente de correlación múltiple & 0,92375677 \\
\hline Coeficiente de determinación $R^{\wedge} 2$ & 0,85332658 \\
\hline$R^{\wedge}$ 2 ajustado & 0,83761157 \\
\hline Error típico & 2337,07305 \\
\hline Observaciones & 32 \\
\hline
\end{tabular}

13 Fuente: Elaboración de los autores a partir de datos de la investigación.

Donde: $\mathrm{E}=$ Valor de los recursos propios o patrimonio; $\mathrm{D}=$ Valor de la deuda; $\mathrm{DB}=$ Valor de la deuda en bonos; $\mathrm{V}=\mathrm{E}+\mathrm{D}=$ valor total de la firma; $\mathrm{E} / \mathrm{E}+\mathrm{D}+\mathrm{DB}=$ Proporción del patrimonio en el valor total de la firma; $\mathrm{D} / \mathrm{E}+\mathrm{D}+\mathrm{DB}=\mathrm{Proporción} \mathrm{de} \mathrm{la}$ deuda en el valor total de la firma; $\mathrm{DB} / \mathrm{E}+\mathrm{D}+\mathrm{DB}=$ Proporción de la deuda en bonos en el valor total de la firma; Ks = Costo del patrimonio en pesos; $\mathrm{Kd}=$ Costo de la deuda; Kbonos = Costo de la deuda en bonos.

14 Fuente: Elaboración de los autores a partir de datos de la investigación. 
Tabla 4. Estimación coeficientes de riesgo

\begin{tabular}{|l|l|l|l|l|l|}
\cline { 2 - 6 } \multicolumn{1}{c|}{} & \multicolumn{4}{c|}{ ANÁLISIS DE VARIANZA } \\
\cline { 2 - 6 } \multicolumn{1}{c|}{} & \multicolumn{1}{c|}{$\begin{array}{c}\text { Grados de li- } \\
\text { bertad }\end{array}$} & Suma de cuadrados & $\begin{array}{c}\text { Promedio de } \\
\text { los cuadrados }\end{array}$ & F & $\begin{array}{c}\text { Valor crítico } \\
\text { de F }\end{array}$ \\
\hline Regresión & 3 & 889746822 & 296582274 & 54,3000984 & $8,5867 \mathrm{E}-12$ \\
\hline Residuos & 28 & 152933492 & 5461910,43 & & \\
\hline Total & 31 & 1042680314 & & & \\
\hline
\end{tabular}

\begin{tabular}{|l|l|l|l|l|l|l|l|l|}
\cline { 2 - 9 } \multicolumn{1}{c|}{} & Coeficientes & Error típico & Estadístico t & Probabilidad & Inferior 95\% & Superior 95\% & $\begin{array}{c}\text { Inferior } \\
\mathbf{9 5 , 0 \%}\end{array}$ & $\begin{array}{c}\text { Superior } \\
\mathbf{9 5 , 0} \%\end{array}$ \\
\hline Intercepción & $-4878,96248$ & 3230,22342 & $-1,51041022$ & 0,14214276 & $-11495,7752$ & 1737,85024 & $-11495,7752$ & 1737,85024 \\
\hline PIB & 0,20445954 & 0,0211794 & 9,65369979 & $2,0833 E-10$ & 0,16107551 & 0,24784357 & 0,16107551 & 0,24784357 \\
\hline DTF & $-107756,216$ & 55177,7834 & $-1,95289135$ & 0,06089367 & $-220782,781$ & 5270,3498 & $-220782,781$ & 5270,3498 \\
\hline INFLACION & 42282,1306 & 62010,5908 & 0,68185337 & 0,50093341 & $-84740,8065$ & 169305,068 & $-84740,8065$ & 169305,068 \\
\hline
\end{tabular}

Tabla 5. Resultados de la estimación de las primas de riesgo modelo APT ${ }^{15}$

\begin{tabular}{|l|c|}
\hline \multicolumn{2}{|c|}{ Estadísticas de la regresión } \\
\hline Coeficiente de correlación múltiple & 0,2300475 \\
\hline Coeficiente de determinación $R^{\wedge} 2$ & 0,05292185 \\
\hline$R^{\wedge} 2$ ajustado & $-0,04855081$ \\
\hline Error típico & 0,13237362 \\
\hline Observaciones & 32 \\
\hline
\end{tabular}

\begin{tabular}{|l|c|c|c|c|c|}
\cline { 2 - 6 } \multicolumn{1}{c|}{} & \multicolumn{5}{c|}{ ANÁLISIS DE VARIANZA } \\
\cline { 2 - 6 } \multicolumn{1}{c|}{} & $\begin{array}{c}\text { Grados de } \\
\text { libertad }\end{array}$ & $\begin{array}{c}\text { Suma de } \\
\text { cuadrados }\end{array}$ & $\begin{array}{c}\text { Promedio de los } \\
\text { cuadrados }\end{array}$ & F & $\begin{array}{c}\text { Valor crítico } \\
\text { de F }\end{array}$ \\
\hline Regresión & 3 & 0,02741638 & 0,00913879 & 0,52153806 & 0,67097866 \\
\hline Residuos & 28 & 0,49063774 & 0,01752278 & & \\
\hline Total & 31 & 0,51805412 & & & \\
\hline
\end{tabular}

\begin{tabular}{|c|c|c|c|c|c|c|c|c|}
\hline & Coeficientes & Error típico & Estadístico t & Probabilidad & Inferior 95\% & Superior $95 \%$ & $\begin{array}{l}\text { Inferior } \\
95,0 \%\end{array}$ & $\begin{array}{c}\text { Superior } \\
95,0 \%\end{array}$ \\
\hline Intercepción & 0,06343599 & 0,04034178 & 1,57246383 & 0,12707609 & $-0,0192004$ & 0,14607237 & $-0,0192004$ & 0,14607237 \\
\hline PRIMA PIB & $-0,00452886$ & 0,00682382 & $-0,66368374$ & 0,51232083 & $-0,01850682$ & 0,00944911 & $-0,01850682$ & 0,00944911 \\
\hline PRIMA INTERES & $-0,20255706$ & 0,28811616 & $-0,70303958$ & 0,4878359 & $-0,79273626$ & 0,38762213 & $-0,79273626$ & 0,38762213 \\
\hline PRIMA INFLACION & 0,73544535 & 3,20418424 & 0,22952655 & 0,82012813 & $-5,82802854$ & 7,29891923 & $-5,82802854$ & 7,29891923 \\
\hline
\end{tabular}

Como se puede observar en Tabla 5, ninguna prima es significativa, por lo que el modelo de las variables macroeconómicas se rechaza para Almacenes Éxito S.A.

15 Fuente: Elaboración de los autores a partir de datos de la investigación. 


\subsection{Costo de los bonos}

En las Tabla 6, 7 y 8 se presentan los costos de cada bono del tipo A, B y C, además del promedio de los bonos emitidos por Almacenes Éxito S.A. (Tabla 9).

Tabla 6. Costo bono A -Almacenes Éxito S.A. ${ }^{16}$

\begin{tabular}{|c|c|c|c|c|c|c|}
\hline \multirow{2}{*}{ Año } & \multirow{2}{*}{ DTF } & \multirow{2}{*}{$\mathbf{2 , 5 \% T A}$} & \multicolumn{2}{|c|}{ Conversión a tasa nominal } & \multirow{2}{*}{ Total NaTV } & \multirow{2}{*}{ Efectiva anual } \\
\cline { 4 - 5 } & & & DTF NaTV & $2,5 \%$ NaTV & & \\
\hline 2006 & $6,27 \%$ & $2,50 \%$ & $6,13 \%$ & $2,52 \%$ & $8,64 \%$ & $8,93 \%$ \\
\hline 2007 & $8,01 \%$ & $2,50 \%$ & $7,78 \%$ & $2,52 \%$ & $10,30 \%$ & $10,70 \%$ \\
\hline 2008 & $9,74 \%$ & $2,50 \%$ & $9,40 \%$ & $2,52 \%$ & $11,92 \%$ & $12,46 \%$ \\
\hline 2009 & $6,15 \%$ & $2,50 \%$ & $6,01 \%$ & $2,52 \%$ & $8,53 \%$ & $8,81 \%$ \\
\hline 2010 & $3,66 \%$ & $2,50 \%$ & $3,61 \%$ & $2,52 \%$ & $6,13 \%$ & $6,27 \%$ \\
\hline
\end{tabular}

Tabla 7. Costo bono B -Almacenes Éxito S.A. ${ }^{17}$

\begin{tabular}{|c|c|c|c|}
\hline Año & IPC & 6\%EA & Costo \\
\hline 2006 & $4,48 \%$ & $6 \%$ & $10,75 \%$ \\
\hline 2007 & $5,69 \%$ & $6 \%$ & $12,04 \%$ \\
\hline 2008 & $7,67 \%$ & $6 \%$ & $14,14 \%$ \\
\hline 2009 & $2,00 \%$ & $6 \%$ & $8,12 \%$ \\
\hline 2010 & $3,17 \%$ & $6 \%$ & $9,36 \%$ \\
\hline
\end{tabular}

Tabla 8. Costo bono C-Almacenes Éxito S.A. ${ }^{18}$

\begin{tabular}{|c|c|}
\hline Año & Costo \\
\hline 2006 & $12,50 \%$ \\
\hline 2007 & $12,50 \%$ \\
\hline 2008 & $12,50 \%$ \\
\hline 2009 & $12,50 \%$ \\
\hline 2010 & $12,50 \%$ \\
\hline
\end{tabular}

Tabla 9. Promedio costo de bonos-Almacenes Éxito S.A. ${ }^{19}$

\begin{tabular}{|c|c|}
\hline Año & Costo \\
\hline 2006 & $10,72 \%$ \\
\hline 2007 & $11,75 \%$ \\
\hline 2008 & $13,03 \%$ \\
\hline 2009 & $9,81 \%$ \\
\hline 2010 & $9,38 \%$ \\
\hline
\end{tabular}

\subsection{Cálculo de las tasas impositivas}

El cálculo de las tasas impositivas se muestra en las tablas 10 y 11.

Tabla 10. Cálculo de las tasas efectivas y marginale ${ }^{20}$

\begin{tabular}{|l|c|c|c|}
\hline & $\mathbf{2 0 0 5}$ & $\mathbf{2 0 0 6}$ & Variaciones \\
\hline Impuestos pagados & 43.056 .148 & 28.007 .483 & -15.048 .665 \\
\hline Utilidad antes de impuestos & 110.903 .849 & 151.183 .530 & 40.279 .681 \\
\hline Impuestos por pagar & 35.348 .133 & 42.335 .764 & 6.987 .631 \\
\hline \multirow{2}{*}{} & & Tasa efectiva & $\mathbf{2 3 , 1 5 \%}$ \\
\cline { 3 - 4 } & & Tasa marginal \\
\cline { 3 - 4 }
\end{tabular}

16 Fuente: Elaboración de los autores a partir de datos de la investigación.

17 Fuente: Elaboración de los autores a partir de datos de la investigación.

18 Fuente: Elaboración de los autores a partir de datos de la investigación.

19 Fuente: Elaboración de los autores a partir de datos de la investigación.

Donde: $D T F=$ Depósito a término fijo; $I P C=$ Índice de precios al consumidor; $T A=$ Trimestre Anticipado; EA = Efectiva anual; naTV

$=$ Nominal anual trimestre vencido.

20 Fuente: Elaboración de los autores a partir de datos de la investigación. 


\begin{tabular}{|c|c|c|c|}
\hline & 2006 & 2007 & Variaciones \\
\hline Impuestos pagados & 28.007 .483 & 40.139 .519 & 12.132 .036 \\
\hline Utilidad antes de impuestos & 151.183 .530 & 171.131 .586 & 19.948 .056 \\
\hline Impuestos por pagar & 42.335 .764 & 46.669 .312 & 4.333 .548 \\
\hline & & \multicolumn{2}{|l|}{ Tasa efectiva } \\
\hline & & \multicolumn{2}{|l|}{ Tasa marginal } \\
\hline & 2007 & 2008 & Variaciones \\
\hline Impuestos pagados & 40.139 .519 & 4.894 .428 & -35.245 .091 \\
\hline Utilidad antes de impuestos & 171.131 .586 & 158.216 .920 & 256.129 .086 \\
\hline Impuestos por pagar & 46.669 .312 & 44.429 .824 & -2.239 .488 \\
\hline & & \multicolumn{2}{|l|}{ Tasa efectiva } \\
\hline & & \multicolumn{2}{|l|}{ Tasa marginal } \\
\hline & 2008 & 2009 & Variaciones \\
\hline Impuestos pagados & 4.894 .428 & 9.882 .567 & 4.988 .139 \\
\hline Utilidad antes de impuestos & 158.216 .920 & 157.184 .982 & 260.546 .404 \\
\hline Cambio Impuestos por pagar & 44.429 .824 & 60.441 .541 & 16.011 .717 \\
\hline & & \multicolumn{2}{|l|}{ Tasa efectiva } \\
\hline & & \multicolumn{2}{|l|}{ Tasa marginal } \\
\hline & 2009 & 2010 & Variaciones \\
\hline Impuestos pagados & 9.882 .567 & 29.971 .800 & 20.089 .233 \\
\hline Utilidad antes de impuestos & 157.184 .982 & 284.429 .147 & 134.811 .164 \\
\hline Impuestos por pagar & 60.441 .541 & 59.837 .864 & -603.677 \\
\hline & & \multicolumn{2}{|l|}{ Tasa efectiva } \\
\hline & & \multicolumn{2}{|l|}{ Tasa marginal } \\
\hline
\end{tabular}

Tabla 11. Resumen tasas efectivas y tasas marginales ${ }^{21}$

\begin{tabular}{|c|c|c|c|}
\hline Años & Tasa Nominal & Tasa efectiva & Tasa marginal \\
\hline 2006 & $35 \%$ & $23,15 \%$ & $17,35 \%$ \\
\hline 2007 & $34 \%$ & $25,99 \%$ & $82,54 \%$ \\
\hline 2008 & $33 \%$ & $3,09 \%$ & $0,00 \%$ \\
\hline 2009 & $33 \%$ & $16,47 \%$ & $8,06 \%$ \\
\hline 2010 & $33 \%$ & $10,54 \%$ & $14,90 \%$ \\
\hline
\end{tabular}

\subsection{Aplicación de los impuestos al costo accionario y costo de capital}

Para los períodos en que la empresa tiene deuda el costo accionario aumenta, debido al mayor riesgo que corren los accionistas, para los años en que la empresa disminuye en gran proporción la deuda o no existe el costo accionario disminuye sustancialmente.

21 Fuente: Elaboración de los autores a partir de datos de la investigación. 
Tabla 12. Costo de capital con impuestos nominales ${ }^{22}$

\begin{tabular}{|l|c|c|c|c|c|}
\hline \multirow{2}{*}{$\begin{array}{l}\text { Variables e } \\
\text { indicadores }\end{array}$} & \multicolumn{5}{|c|}{ Años } \\
\cline { 2 - 6 } & $\mathbf{2 0 0 6}$ & $\mathbf{2 0 0 7}$ & $\mathbf{2 0 0 8}$ & $\mathbf{2 0 0 9}$ & $\mathbf{2 0 1 0}$ \\
\hline$D / E$ & $42,41 \%$ & $60,09 \%$ & $30,04 \%$ & $12,38 \%$ & $0,00 \%$ \\
\hline DB/E & $17,51 \%$ & $6,61 \%$ & $6,61 \%$ & $4,58 \%$ & $9,80 \%$ \\
\hline Ko & $20,44 \%$ & $21,14 \%$ & $23,04 \%$ & $18,89 \%$ & $13,75 \%$ \\
\hline$t$ & $35,00 \%$ & $34,00 \%$ & $33,00 \%$ & $33,00 \%$ & $33,00 \%$ \\
\hline $1-t$ & 0,65 & $66,00 \%$ & $67,00 \%$ & $67,00 \%$ & $67,00 \%$ \\
\hline Kd & $9,41 \%$ & $10,08 \%$ & $11,30 \%$ & $11,60 \%$ & $11,60 \%$ \\
\hline Kbonos & $10,72 \%$ & $11,75 \%$ & $13,03 \%$ & $9,81 \%$ & $9,38 \%$ \\
\hline Ko - Kd & $11,03 \%$ & $11,06 \%$ & $11,74 \%$ & $7,29 \%$ & $2,15 \%$ \\
\hline Ko-Kbonos & $9,72 \%$ & $9,39 \%$ & $10,01 \%$ & $9,08 \%$ & $4,37 \%$ \\
\hline Kd(1-t) & $6,12 \%$ & $6,65 \%$ & $7,57 \%$ & $7,77 \%$ & $7,77 \%$ \\
\hline Kbonos(1-t) & $6,97 \%$ & $7,76 \%$ & $8,73 \%$ & $6,57 \%$ & $6,28 \%$ \\
\hline Ks & $24,58 \%$ & $25,94 \%$ & $25,85 \%$ & $19,77 \%$ & $14,03 \%$ \\
\hline CK & $17,76 \%$ & $18,27 \%$ & $21,00 \%$ & $17,99 \%$ & $13,34 \%$ \\
\hline
\end{tabular}

El costo de capital en los años 2006 a 2009 aumento con el efecto de los impuestos (Tabla 12), siendo contrario a lo que se esperaba, por el alto nivel de apalancamiento que poseía la empresa en ese momento. Esto hizo que el riesgo aumentara y que el costo accionario también lo hiciera, pero en mayor proporción, no siendo compensado por el beneficio tributario de la deuda. Para el año 2010, disminuye el costo de capital con el efecto de los impuestos, compensado por el beneficio tributario. De esta manera, el costo accionario aumenta en mayor proporción que con los impuestos nominales porque las tasas efectivas son más bajas.

El costo de capital también aumenta en mayor proporción con las tasas efectivas en prácticamente todo el período de estudio con el efecto de los impuestos efectivos debido a que la tasa de impuestos es más baja que la nominal y no alcanza a ser compensado por el beneficio tributario en los períodos que hay deuda (Tabla 13).

\section{Conclusiones}

En el año 2008, la empresa obtuvo un beta alto causado por un mayor riesgo del mercado como consecuencia de la crisis financiera mundial, luego en los años 2009 y 2010 se observó una disminución del riesgo debido a la superación de la crisis.

Los factores comunes utilizados en la metodología APT fueron las variables macroeconómicas, PIB, Inflación y tasa de interés, de las cuales el PIB fue la única variable que explicó el comportamiento del costo accionario. Al estimar las primas de riesgo ninguna resulto significativa. Por lo que la metodología APT utilizando las variables macroeconómicas se rechaza como un todo para los datos analizados. Al presentar los estimativos del costo accionario con el coeficiente del PIB, los resultados son más altos que en el modelo CAPM, permitiendo observar el efecto que tiene la situación económica del país en las rentabilidades de la empresa analizada.

22 Fuente: Elaboración de los autores a partir de datos de la investigación.

Donde: $E=$ Valor de los recursos propios o patrimonio; $D=$ Valor de la deuda; $D B=$ Valor de la deuda en bonos; $D / E=$ Proporción de la deuda en el valor total del patrimonio; $D B / E$ = Proporción de la deuda de los bonos en el valor total de la firma; Ko = costo del patrimonio de una empresa sin deuda; $K s=$ Costo del patrimonio de una empresa con deuda; $K d=$ Costo de la deuda; $K b o n o s=$ costo de la deuda en bonos; $t=$ Tasa impositiva; $C K=$ Costo de capital. 
Tabla 13. Costo de capital con impuestos efectivos ${ }^{23}$

\begin{tabular}{|c|c|c|c|c|c|}
\hline \multirow{2}{*}{$\begin{array}{r}\text { Variables e } \\
\text { indicadores }\end{array}$} & \multicolumn{5}{|c|}{ Años } \\
\hline & 2006 & 2007 & 2008 & 2009 & 2010 \\
\hline$D / E$ & $42,41 \%$ & $60,09 \%$ & $30,04 \%$ & $12,38 \%$ & $0,00 \%$ \\
\hline$D B / E$ & $17,51 \%$ & $6,61 \%$ & $6,61 \%$ & $4,58 \%$ & $9,80 \%$ \\
\hline Ko & $20,44 \%$ & $21,14 \%$ & $23,04 \%$ & $18,89 \%$ & $13,75 \%$ \\
\hline t & $23,15 \%$ & $25,99 \%$ & $3,09 \%$ & $16,47 \%$ & $10,54 \%$ \\
\hline $1-t$ & 0,77 & 0,74 & 0,97 & 0,8353 & 0,8946 \\
\hline$K d$ & $9,41 \%$ & $10,08 \%$ & $11,30 \%$ & $11,60 \%$ & $11,60 \%$ \\
\hline Kbonos & $10,72 \%$ & $11,75 \%$ & $13,03 \%$ & $9,81 \%$ & $9,38 \%$ \\
\hline$K o-K d$ & $11,03 \%$ & $11,06 \%$ & $11,74 \%$ & $7,29 \%$ & $2,15 \%$ \\
\hline Ko - Kbonos & $9,72 \%$ & $9,39 \%$ & $10,01 \%$ & $9,08 \%$ & $4,37 \%$ \\
\hline$K d(1-t)$ & $7,23 \%$ & $7,46 \%$ & $10,95 \%$ & $9,69 \%$ & $10,38 \%$ \\
\hline Kbonos(1-t) & $8,24 \%$ & $8,70 \%$ & $12,63 \%$ & $8,19 \%$ & $8,39 \%$ \\
\hline Ks & $25,34 \%$ & $26,52 \%$ & $27,10 \%$ & $19,99 \%$ & $14,13 \%$ \\
\hline Ck & $18,66 \%$ & $18,94 \%$ & $22,85 \%$ & $18,44 \%$ & $13,62 \%$ \\
\hline
\end{tabular}

Al replantear la proposición I de Modigliani \& Miller (1958) con la proposición I de Modigliani \& Miller (1963), se infiere que a medida que una empresa se endeuda, su valor aumenta debido a que el ahorro fiscal obtenido es mayor. Para el caso Almacenes Éxito S.A. en el período de estudio se pudo observar que los mayores beneficios tributarios obtenidos fueron por tratamientos tributarios especiales y no por la financiación con deuda, lo cual fue mínimo. Entre los tratamientos tributarios especiales estuvo por ejemplo la Ley 1111 de 2006, de donde se deducen las bajas tasas efectivas calculadas. Los beneficios tributarios obtenidos por la empresa estudiada han sido en parte el resultado de su estrategia de expansión de activos, la cual fue desarrollada en el período analizado, siendo la adquisición de Carulla - Vivero la más significativa.

La Proposición II de Modigliani \& Miller (1958) relaciona directamente el rendimiento esperado sobre el capital social de una firma apalancada con el nivel de endeudamiento, haciendo que el costo de los recursos propios aumente, debido al mayor riesgo en que se incurre. Se anulan las economías obtenidas por el ma- yor valor de deuda y el costo del capital se mantiene constante. Al incluir los impuestos con la proposición de Modigliani \& Miller (1963) se concluye que a medida que se incrementa la financiación por deuda, el costo de los recursos propios se incrementa en menor proporción que el incremento de la deuda, y el costo de la deuda se ve disminuido por la tasa impositiva producto de la deducción de impuestos. Para el caso Almacenes Éxito S.A. al incluir los impuestos en el costo de los recursos propios y recursos obtenidos por deuda, no disminuye el costo de capital debido al nivel de apalancamiento que posee la empresa para los datos estudiados. Esto hizo que el riesgo aumentase, y por lo tanto, el costo accionario también lo hiciera en mayor proporción, alcanzando a ser compensado por el beneficio tributario de la deuda.

Para dar respuesta a la pregunta del presente estudio: ¿cuál es el efecto de los impuestos corporativos en el costo de capital del caso Almacenes Éxito S.A. período 2006 - 2010? Se encuentra que el efecto de los impuestos corporativos en el costo de capital de Almacenes Éxito S.A. período 2006 - 2010 no genera una disminución en el costo de capital.

23 Fuente: Elaboración de los autores a partir de datos de la investigación. 
Entonces, la empresa no tiene un beneficio en el costo de capital obtenido de la financiación con deuda, por lo que el incentivo para ésta de buscar financiación con deuda, representa sus decisiones de inversión. Como se pudo observar, con su estrategia de expansión en infraestructura comercial, incrementando la rentabilidad y flujos de caja.

Lo anterior, se podría decir que es coherente con la Proposición I de Modigliani \& Miller (1958) que dice: "el valor de la empresa depende de sus rendimientos esperados descontados a una tasa $k$ y no a su estructura de financiación". En otras palabras, Almacenes Éxito S.A. está aumentando su valor, principalmente desde los rendimientos esperados $y$ no desde la disminución de la tasa $k$.

Almacenes Éxito S.A. a medida que aumenta su financiación con recursos propios, el costo de capital disminuye, debido a que el riesgo representado en el coeficiente beta disminuye por la favorable situación de la economía, y también disminuye la tasa libre de riesgo obteniéndose un costo de los recursos propios bastante bajo, convirtiéndose en un recurso no tan $\operatorname{costoso}^{24}$. Lo que posiblemente podría explicar sus continuas financiaciones con acciones. Además, parece que la dirección de la empresa tiene cierta aversión al riesgo generado por la deuda, ya que en uno de sus informes, dice el gerente: "queremos lograr la mayor rentabilidad pero con prudencia", lo cual podría ser causado por la gran relación que tiene la organización con la situación económica del país.

En últimas, el presente estudio es un aporte a los estudios de costo de capital, ya que trata sobre uno de los determinantes más importantes del costo de capital como lo son los impuestos, y del cual, la única referencia encontrada es la de Herrera \& Mora (1998).

Por otro lado, el método de caso permitió observar la forma cómo se libera caja y se mejoran los flujos de efectivo, sin ir en detrimento de la operación de la empresa, aumentando la rentabilidad operativa y disminuyendo la necesidad de financiación externa.
En cuanto a la metodología APT, se puede decir que su principal ventaja fue que permitió incluir otros tipos de riesgo sistemático diferentes al del mercado. Una de sus limitantes fue que los factores de estos otros riesgos sistemáticos no estaban definidos, por la existencia de diferentes versiones dependiendo de cuál tiene mayor capacidad para reproducir el comportamiento de la rentabilidad de los activos.

\section{Referencias}

Clavijo, S. (2005). "Tributación, equidad y eficiencia en Colombia: guía para salir de un sistema tributario amalgamado". Documento de trabajo. Banco de la República. Disponible en: http:// www.banrep.gov.co/junta/publicaciones/Clavijo/tribu1204.pdf

Decreto 624 de 1989. "Por el cual se expide el Estatuto Tributario de los Impuestos Administrados por la Dirección General de Impuestos Nacionales". Presidente de la República de Colombia. Disponible en: http:/www.cancilleria.gov.co/sites/default/files/ tramites servicios/pasaportes/decreto-624-1989.pdf

García, O. (2003). Valoración de empresas, gerencia del valor y EVA. Cali: Prensa Moderna Impresores S.A.

Gitman, L. (2007). Principios de Administración Financiera. México. Pearson.

Fuentes, R., Gregoire, J. \& Zurita, S. (2005) "Factores Macroeconómicos en Retornos Accionarios Chilenos". Banco Central de Chile, Documentos de Trabajo $\mathrm{N}^{\circ} 316$.

Herrera, S. \& Mora, H. (1998). El costo de capital en las empresas colombianas y el efecto de la tributación. Superintendencia de Valores. Antares Editores S.A, Bogotá.

Modigliani, F. \& Miller, M. (1958) "The Cost of Capital, and H. Miller Corporation Finance and the Theory of Investment". En: The American Economic Review, 48 (3).

Modigliani, F. \& Miller, M. (1963) "Corporate Income Taxes and the Cost of Capital: A Correction". En: The American Economic Review, 53.

Ley 1111 de 2006. Por la cual se modifica el estatuto tributario de los impuestos administrados por la Dirección de Impuestos y Aduanas Nacionales. Congreso Nacional. Disponible en: http://www.secretariasenado.gov.co/senado/basedoc/ley/2006/ ley_11112006.html

Ross, S. (1976) "The Arbitrage of Capital Asset Pricing". En: Journal of Economic Theory, 13 (2).

Ross, S., Westerfield, R. \& Jaffe, J. (2003). Finanzas Corporativas. México: Mc Graw Hill.

Ryan, B., Scapen, R. \& Theobald, M. (2004) Metodología de la investigación en Finanzas y Contabilidad. (Cap. 8: Estudios de casos). Barcelona: Ediciones Deusto.

Salas, R., Gutiérrez, J. \& Vélez, I. (2011). Valor de los ahorros en impuestos por deuda en Colombia: un estudio empírico. Documentos de trabajo, Universidad Tecnologica de Bolivar. Disponible en: http://papers.ssrn.com/sol3/papers.cfm?abstract_ id $=1899685$

24 Teniendo en cuenta una de las limitaciones de la Metodología CAPM en la que tiende a sobrevalorar el costo de los recursos propios cuando el coeficiente Beta es mayor a 1 y a subvalorarlo cuando es menor que 1. 


\section{ANEXO 1}

\section{Derivación de formulas ${ }^{25}$}

$$
\begin{aligned}
& U A I I_{V L}=U A I I\left(1-T_{C}\right)+T_{c} K_{d} D, \text { o sea que } \\
& U A I I_{V L}-T_{C} K_{d} D=U A I I\left(1-T_{C}\right) \text { si se reemplaza en: } \\
& V_{L}=\frac{U A I I_{V L}\left(1-T_{C}\right)}{K_{O}}+\frac{T_{C} K_{d} D}{K_{d}}
\end{aligned}
$$

Si ambos términos se multiplican por $\mathrm{K}_{\mathrm{o}} \mathrm{y}$ se dividen por $\mathrm{V}_{\mathrm{L}}$ se llega a que el costo de capital medio ponderado es igual a:

$\frac{U A I I_{V L}}{V_{L}}=K_{O}-T_{C}\left(K_{O}-K_{d}\right) \frac{D}{V_{L}}$

Si se toma la ecuación anterior (Proposición I) y se reemplaza UAIIVL por UODI $+K_{d} D$ y $\mathrm{V}_{\mathrm{L}}$ por $\mathrm{S}_{\mathrm{L}}+\mathrm{D}_{\mathrm{L}}$, se encuentra que el costo de capital propio es igual a:

$\frac{U O D I}{S_{L}}=K_{O}+\left(1-T_{C}\right)\left(K_{O}-K_{d}\right) \frac{D_{L}}{S_{L}}$ donde,

$\frac{U O D I}{S_{L}}=$ es el costo de capital propio de una empresa apalancada

Entonces,

$k_{s}=k_{o}+\frac{D}{E}\left(1-T_{c}\right)\left(k_{o}-k_{d}\right)$

\footnotetext{
Fuente: Elaboración de los autores
} 


\section{ANEXO $2^{26}$}

Balance General Almacenes Éxito S.A. (Miles de pesos)

\begin{tabular}{|c|c|c|c|c|c|}
\hline & 2.006 & 2.007 & 2008 & 2009 & 2010 \\
\hline Caja & 21.873 .496 & 34.475 .221 & 79.368 .817 & 51.607 .679 & 64.940 .627 \\
\hline Bancos & 97.784 .631 & 80.833 .896 & 40.956 .362 & 149.519 .805 & 44.547 .804 \\
\hline Cuentas de ahorro & 6.356 .553 & 4.629 .800 & 61.199 .498 & 192.188 .118 & 404.898 .234 \\
\hline SUBTOTAL DISPONIBLE & 126.014 .680 & 161.607.197 & 181.524.677 & 392.315 .602 & 514.386 .665 \\
\hline INVERSIONES A CORTO PLAZO & 106.484 .671 & 341.926 .811 & 220.528 .000 & 240.626 .336 & 274.451 .927 \\
\hline Clientes & 48.776 .324 & 52.919 .458 & 50.042 .785 & 61.446 .876 & 36.426 .134 \\
\hline Cuentas por cobrar a Casa Matriz & & 23.646 & 71.592 & 71.592 & 71.592 \\
\hline Cuentas por cobrar a Vincu.Económicos & & 69.988 .806 & 10.871 .259 & 9.930 .023 & 21.674 .692 \\
\hline Anticipos y avances & 1.113 .638 & 1.560 .997 & 824.112 & 390.364 & 1.078 .329 \\
\hline Ingresos por cobrar CP & 6.126 .539 & 7.160 .501 & 8.443 .329 & 13.319 .157 & 14.781 .036 \\
\hline AnticImptos y Contrib o Saldos a favor & 1.492 .897 & 42.196 .324 & 49.408 .538 & 59.533 .990 & 57.534 .216 \\
\hline Reclamaciones (CP) & 1.903 & 86.245 .370 & 36.930 .434 & & \\
\hline Cuentas x Cobrar a trabajadores(CP) & 441.090 & 1.154 .366 & 1.616 .434 & 4.683 .774 & 2.610 .441 \\
\hline Deudores varios (CP) & 39.685 .730 & 43.945 .752 & 90.106 .746 & 81.663 .449 & 293.546 .591 \\
\hline provisiones (CP) & 3.263 .042 & 5.082 .170 & 7.989 .335 & 7.754 .891 & 72.396 .025 \\
\hline SUBTOTAL DEUDORES CORTO PLAZO & 94.375 .079 & 300.113 .050 & 240.325 .894 & 223.284.334 & 355.327 .006 \\
\hline Materias Primas & & & & & 3.050 .837 \\
\hline Productos en Proceso & & & & & 1.430 .936 \\
\hline Productos Terminados & & & & & 1.022 .235 \\
\hline Mcias no Fabricadas por la empresa & 507.021 .529 & 719.018 .372 & 886.770 .443 & 778.522 .000 & 807.192 .558 \\
\hline Materiales Repuestos y accesorios & 5.924 .382 & 7.641 .074 & 14.700 .693 & 15.272 .968 & 21.757 .456 \\
\hline Envases y empaques & & & & & 2.335 .666 \\
\hline Inventarios en Tránsito & 19.721 .347 & 21.731 .215 & 30.827 .361 & 13.508 .763 & 19.257 .349 \\
\hline provisiones $(\mathrm{CP})$ & 12.892 .901 & 16.422 .912 & 19.279 .061 & 21.666 .940 & 33.438 .998 \\
\hline SUBTOTAL INVENTARIOS & 519.774 .357 & 731.967.749 & 913.019.436 & 785.636 .791 & 822.608 .039 \\
\hline Gastos pagados xAnticipado CP & 10.824 .252 & 34.836 .156 & 7.821 .412 & 7.821 .278 & 11.444 .772 \\
\hline Cargos Diferidos CP & 19.682 .273 & 34.373 .068 & 47.381 .346 & 18.731 .874 & 30.149 .570 \\
\hline SUBTOTAL DIFERIDOS & 30.506 .525 & 69.209 .224 & 55.202 .758 & 26.553 .152 & 41.594 .342 \\
\hline TOTAL ACTIVO CORRIENTE & 877.155 .312 & 1.604 .824 .031 & 1.610 .600 .765 & 1.668.416.215 & 2.008.367.979 \\
\hline INVERSIONES LP & 220.173.511 & 633.723 .614 & 704.891 .779 & 936.731 .847 & 172.007 .593 \\
\hline anticipos y Avances (LP) & 7.994 .228 & 18.302 .265 & 7.468 .240 & 10.088 .010 & 6.718 .683 \\
\hline Promesa de Compraventa (LP) & 5.580 .219 & 5.531 .650 & 13.017 .447 & 14.668 .496 & 10.883 .351 \\
\hline Deudores varios (LP) & 35.507 .228 & 35.315 .673 & 37.025 .724 & 34.184 .858 & 33.971 .329 \\
\hline SUBTOTAL DEUDORES A LARGO PLAZO & 49.081 .675 & 59.149 .588 & 57.511 .411 & 58.941 .364 & 51.573 .363 \\
\hline
\end{tabular}

26 Fuente: Superintendencia de Sociedades. Extraídos de: http://www.supersociedades.gov.co/ 


\begin{tabular}{|c|c|c|c|c|c|}
\hline PROPIEDADES PLANTA Y EQUIPO NETO & 1.638.855.718 & 1.597.026.264 & 1.872 .404 .388 & 1.793.714.193 & 2.050.849.594 \\
\hline Crédito Mercantil & 8.398 .654 & 698.139 .583 & 698.446 .318 & 1.006 .658 .885 & 1.021.114.370 \\
\hline Marcas & 3.417 .195 & 3.460 .695 & 3.460 .695 & 3.460 .695 & 32.363 .247 \\
\hline Concesiones y franquicias & 685.452 & 685.452 & 685.452 & & \\
\hline Derechos & 3.891 .698 & 95.135 .201 & 103.274 .651 & 29.000 .916 & 54.619 .325 \\
\hline Amortización acumulada & 10.584 .275 & 38.902 .795 & 60.027 .919 & 62.314 .602 & 103.164.026 \\
\hline SUBTOTAL INTANGIBLES & 5.808 .724 & 758.518 .136 & 745.839.197 & 976.805 .894 & 1.004.932.916 \\
\hline Gastos pagados xAnticipado LP & & 10.368.392 & 10.356 .556 & 9.467 .728 & 8.578 .900 \\
\hline Cargos Diferidos LP & 80.872 .311 & 112.962 .291 & 166.146 .307 & 176.849 .258 & 218.045 .435 \\
\hline Cargos por correcmonet diferida LP & 10.420 .792 & 9.522 .283 & 8.468 .354 & 5.666 .555 & 4.609 .457 \\
\hline SUBTOTAL DIFERIDOS & 91.293 .103 & 132.852 .966 & 184.971.217 & 191.983 .541 & 231.233.792 \\
\hline Bienes de Arte y cultura & 243.006 & 285.331 & 285.331 & 285.331 & 285.331 \\
\hline SUBTOTAL OTROS ACTIVOS & 243.006 & 285.331 & 285.331 & 285.331 & 285.331 \\
\hline De inversiones & $(15.538 .989)$ & $(11.330 .385)$ & 13.449 .188 & 9.525 .889 & 25.872 .052 \\
\hline De propiedades planta y equipo & 733.094 .512 & 811.306 .905 & 747.658 .087 & 755.078 .689 & 1.095.215.735 \\
\hline De otros Activos & & 30.302 .000 & & & \\
\hline SUBTOTAL VALORIZACIONES & 717.555 .523 & 830.278 .520 & 761.107 .275 & 764.604 .578 & 1.121.087.787 \\
\hline TOTAL ACTIVO NO CORRIENTE & 2.723.011.269 & 4.011 .834 .419 & 4.327.010.598 & 4.723 .066 .748 & 4.631.970.376 \\
\hline TOTAL ACTIVO & 3.600 .166 .575 & 5.616 .658 .451 & 5.937.611.362 & 6.391 .482 .963 & 6640338355 \\
\hline OBLIGACIONES FINANCIERAS (CP) & 171.940 .799 & 35.878 .530 & 476.458 .000 & 231.718 .366 & 357.294 .285 \\
\hline PROVEEDORES & 476.531 .884 & 677.576 .325 & 966.139 .765 & 1.040.292.174 & 1.098.496.166 \\
\hline A Compañías vinculadas (CP) & 3.130 .279 & 55.468 .028 & 119.388 .000 & 204.375 .376 & 866.154 \\
\hline A contratistas $(\mathrm{CP})$ & 36.000 .016 & 23.015 .682 & 21.078 .855 & 1.539 .832 & 30.096 .102 \\
\hline Ordenes de compra x Utilizar & 11.057 .621 & 16.819 .010 & 17.685 .671 & 19.442 .517 & 24.297 .253 \\
\hline Costo y Gastos x pagar CP & 102.107.962 & 134.684 .688 & 138.153 .000 & 110.790 .266 & 161.043 .488 \\
\hline Dividendos o participación por pagar & 16.283 .304 & 595.283 & 17.568 .329 & 4.731 .545 & 20.714 .640 \\
\hline Retención en la fuente & 6.555 .014 & 8.613 .169 & 10.883 .939 & 9.713 .778 & 11.061 .031 \\
\hline Impuesto a las ventas retenido & 4.430 .486 & 4.885 .179 & 5.170 .083 & 5.400 .337 & 7.068 .136 \\
\hline Impuesto de industria y comercio retenido & 455.963 & 651.437 & 933.539 & 1.159 .461 & 1.422 .850 \\
\hline Retenciones y aportes de nomina & 7.396 .509 & 6.292 .991 & 7.592 .487 & 11.270 .284 & 15.264 .805 \\
\hline Acreedores varios (CP) & 606.865 & 616.865 & 606.865 & 606.865 & 606.865 \\
\hline SUBTOTAL CUENTAS POR PAGAR CP & 188.024 .019 & 251.632 .332 & 339.060 .768 & 369.030 .170 & 272.441 .324 \\
\hline IMPUESTOS GRAVAMENES Y TASAS & 42.335 .764 & 46.669 .312 & 44.429 .824 & 60.441 .541 & 59.837 .864 \\
\hline OBLIGACIONES LABORALES CP & 31.043 .640 & 26.643 .571 & 28.263 .544 & 31.682 .142 & 46.814 .981 \\
\hline Para Costos y Gastos & 39.953 .359 & 57.225 .796 & 46.142 .331 & 30.743 .696 & 42.522 .011 \\
\hline Para obligaciones laborales & 2.505 .933 & 2.955 .652 & 32.757 & 43.961 & 168.802 \\
\hline Pensiones de jubilación & 4.164 .935 & 3.488 .001 & 3.013 .938 & 2.513 .590 & 3.079 .279 \\
\hline $\begin{array}{l}\text { SUBTOTAL PASIVOS } \\
\text { ESTIMADOS Y PROVISIONES }\end{array}$ & 46.624 .227 & 63.669 .449 & 49.189 .026 & 33.301 .247 & 45.770 .092 \\
\hline Anticipos y avances recibidos CP & 1.620 .030 & 1.933 .264 & 2.089 .577 & 2.171 .971 & 2.341 .906 \\
\hline
\end{tabular}




\begin{tabular}{|c|c|c|c|c|c|}
\hline Depositos recibidos (CP) & 3.923 .619 & 7.770 .535 & 3.282 .003 & 872.607 & 2.838 .665 \\
\hline Ingresos recibidos para terceros (CP) & 6.978 .155 & 3.681 .963 & 8.428 .817 & 9.844 .786 & 6.899 .842 \\
\hline SUBTOTAL OTROS PASIVOS CP & 12.521 .804 & 13.385 .763 & 13.800 .397 & 12.889 .364 & 12.080 .413 \\
\hline Bonos en circulación & & & & & 30.350 .000 \\
\hline SUBTOTAL BONOS Y PAPELES COMERCIALES & & & & & 30.350 .000 \\
\hline TOTAL PASIVO CORRIENTE & 969.022 .137 & 1.115 .455 .282 & 1.917 .341 .324 & 1.779 .355 .004 & 1.923 .085 .125 \\
\hline OBLIGACIONES FINANCIERAS (LP) & 254.363 .930 & 955.290 .250 & 477.506 .539 & 283.663 .944 & \\
\hline A Compañias Vinculadas (LP) & & & 182 & & \\
\hline Costo y Gastos x pagar LP & 16.280 .501 & & 413 & & \\
\hline SUBTOTAL CUENTAS POR PAGAR LP & 16.280 .501 & & 595 & & \\
\hline OBLIGACIONES LABORALES LP & 527.036 & 365.463 & 510.076 & 732.232 & 574.619 \\
\hline Pensiones de jubilación LP & 10.593 .165 & 9.870 .997 & 9.466 .409 & 9.523 .016 & 16.843 .961 \\
\hline $\begin{array}{l}\text { SUBTOTAL PASIVOS } \\
\text { ESTIMADOS Y PROVISIONESLP }\end{array}$ & 10.593 .165 & 9.870 .997 & 9.466 .409 & 9.523 .016 & 16.843 .961 \\
\hline Crédito x Correc. Monetaria Diferida LP & 15.791 .033 & 14.415 .314 & 12.486 .255 & 8.355 .113 & 6.796 .463 \\
\hline Impuesto Diferido (LP) & 3.940 .463 & 31.219 .819 & 2.644 .213 & & 8.371 .227 \\
\hline SUBTOTAL OTROS PASIVOS LP & 19.731 .496 & 45.635 .133 & 15.130 .468 & 8.355 .113 & 15.167 .690 \\
\hline Avances y Anticipos Recibidos (LP) & & & 18.688 .684 & 41.730 .729 & 42.795 .314 \\
\hline SUBTOTAL OTROS PASIVOS LP & & & 18.688 .684 & 41.730 .730 & 42.795 .314 \\
\hline Bonosen circulación (LP) & 105.000 .000 & 105.000 .000 & 105.000 .000 & 105.000 .000 & 224.650 .000 \\
\hline TOTAL PASIVOS NO CORRIENTE & 406.496 .128 & 1.116 .161 .843 & 626.302 .772 & 449.005 .035 & 300.031 .584 \\
\hline TOTAL PASIVO & 1.375.518.265 & 2.231.617.125 & 2.543 .644 .096 & 2.228.360.039 & 2.223.116.709 \\
\hline Capital Suscrito y pagado & 2.095 .630 & 2.842 .630 & 2.842 .630 & 3.338 .098 & 3.339 .695 \\
\hline SUBTOTAL CAPITAL SOCIAL & 2.095 .630 & 2.842 .630 & 2.842 .630 & 3.338 .098 & 3.339 .695 \\
\hline Prima en coloc. Acc. Cuotaso partes de int s & 597.630 .614 & 1.586 .806 .477 & 1.586 .806 .477 & 2.288.213.427 & 2.289 .589 .024 \\
\hline SuperavitMetodo e Particiapción & 1.958 .695 & 20.173 .834 & 34.390 .948 & 40.286 .891 & $(11.070 .048)$ \\
\hline SUBTOTAL SUPERAVIT DE CAPITAL & 599.589 .309 & 1.606 .980 .311 & 1.621.197.425 & 2.328 .500 .318 & 2.278 .518 .977 \\
\hline RESERVAS & 161.298 .946 & 214.386 .842 & 277.398 .389 & 362.560 .360 & 429.901 .013 \\
\hline REVALORIZACION DEL PATRIMONIO & 613.120 .280 & 591.748 .380 & 570.376 .480 & 549.004 .580 & 321.727 .621 \\
\hline RESULTADOS DEL EJERCICIO & 23.176.047 & 130.992 .067 & 153.322.491 & 147.302 .415 & 252.833 .979 \\
\hline UTILIDADES ACUMULADAS & & 7.812 .575 & 7.812 .575 & 7.812 .575 & 7.812 .575 \\
\hline Derechos Contingentes & 1.494 .678 .280 & 1.025 .893 .737 & 831.647 .578 & 435.359 .420 & 257.568 .946 \\
\hline Deudoras Fiscales & 552.597 .699 & 672.574 .837 & 787.059 .697 & 1.192 .664 .155 & 540.891 .818 \\
\hline Deudoras de Control & 1.107 .385 .818 & 1.472 .782 .362 & 1.697 .121 .994 & 2.049 .045 .779 & 2.677 .289 .731 \\
\hline RESULTADOS DE EJERCICIOS ANTERIORES & 7.812 .575 & 7.812 .575 & 7.812 .575 & 7.812 .575 & 7.812 .575 \\
\hline SUPERAVIT POR VALORIZACIONES & 717.555 .523 & 830.278 .521 & 761.107 .276 & 764.604 .578 & 1.121 .087 .787 \\
\hline TOTAL PATRIMONIO & 2.224.648.310 & 3.385 .041 .326 & 3.393 .967 .266 & 4.163.122.924 & 4.417.221.647 \\
\hline TOTAL PASIVO Y PATRIMONIO & 3.600 .166 .575 & 5.616 .658 .451 & 5.937 .611 .362 & 6.391 .482 .963 & 6.640 .338 .356 \\
\hline
\end{tabular}


Estado de Resultados ALMACENES ÉXITO S.A.

\begin{tabular}{|l|c|c|c|c|c|}
\hline & $\mathbf{2 0 0 6}$ & $\mathbf{2 0 0 7}$ & $\mathbf{2 0 0 8}$ & $\mathbf{2 0 0 9}$ & $\mathbf{2 0 1 0}$ \\
\hline Ingresos operacionales & 4.260 .315 .903 & 4.878 .286 .503 & 5.930 .680 .086 & 6.970 .665 .639 & 7.507 .132 .150 \\
\hline MENOS: Costos De Ventas y De Prestación De Servicios & 3.216 .996 .460 & 3.681 .607 .117 & 4.477 .871 .614 & 5.257 .310 .763 & 5.658 .966 .110 \\
\hline UTILIDAD BRUTA & $\mathbf{1 . 0 4 3 . 3 1 9 . 4 4 3}$ & $\mathbf{1 . 1 9 6 . 6 7 9 . 3 8 6}$ & $\mathbf{1 . 4 5 2 . 8 0 8 . 4 7 2}$ & $\mathbf{1 . 7 1 3 . 3 5 4 . 8 7 6}$ & $\mathbf{1 . 8 4 8 . 1 6 6 . 0 4 0}$ \\
\hline MENOS: Gastos Operacionales De Administración & 104.043 .993 & 133.581 .181 & 158.918 .325 & 188.810 .220 & 227.679 .220 \\
\hline MENOS: Gastos Operacionales De Ventas & 770.844 .027 & 855.740 .833 & 1.096 .330 .379 & 1.379 .914 .508 & 1.305 .597 .390 \\
\hline UTILIDAD OPERACIONAL & $\mathbf{1 6 8 . 4 3 1 . 4 2 3}$ & $\mathbf{2 0 7 . 3 5 7 . 3 7 2}$ & $\mathbf{1 9 7 . 5 5 9 . 7 6 8}$ & $\mathbf{1 4 4 . 6 3 0 . 1 4 8}$ & $\mathbf{3 1 4 . 8 8 9 . 4 3 0}$ \\
\hline MAS: IngresOS No Operacionales & 168.377 .816 & 578.332 .004 & 827.961 .656 & 739.533 .400 & 328.089 .890 \\
\hline MENOS: Gastos No Operacionales & 215.160 .246 & 614.557 .790 & 867.304 .504 & 726.978 .566 & 358.550 .173 \\
\hline $\begin{array}{l}\text { UTILIDAD ANTES DE IMPUESTOS } \\
\text { Y AJUSTE POR INFLACION }\end{array}$ & $\mathbf{1 2 1 . 6 4 8 . 9 9 3}$ & $\mathbf{1 7 1 . 1 3 1 . 5 8 6}$ & $\mathbf{1 5 8 . 2 1 6 . 9 2 0}$ & $\mathbf{1 5 7 . 1 8 4 . 9 8 2}$ & $\mathbf{2 8 4 . 4 2 9 . 1 4 7}$ \\
\hline Ajustes Por Inflación & 29.534 .537 & & & & \\
\hline MENOS:Impuestos de Renta y Complementarios & 28.007 .483 & 40.139 .519 & 4.894 .428 & 9.882 .567 & 29.971 .800 \\
\hline GANANCIAS Y PERDIDAS & $\mathbf{1 2 3 . 1 7 6 . 0 4 7}$ & $\mathbf{1 3 0 . 9 9 2 . 0 6 7}$ & $\mathbf{1 5 3 . 3 2 2 . 4 9 2 , 0 0}$ & $\mathbf{1 4 7 . 3 0 2 . 4 1 5}$ & $\mathbf{2 5 4 . 4 5 7 . 3 4 7}$ \\
\hline
\end{tabular}




\section{ANEXO 3}

\section{Estimaciones betas modelo CAPM años 2006 a $2010^{27}$}

Estimación Beta año 2006

\begin{tabular}{|l|c|}
\hline \multicolumn{2}{|c|}{ Estadísticas de la regresión } \\
\hline Coeficiente de correlación múltiple & 0,139625721 \\
\hline Coeficiente de determinación $\mathrm{R}^{\wedge} 2$ & 0,019495342 \\
\hline $\mathrm{R}^{\wedge} 2$ ajustado & 0,00026976 \\
\hline Error típico & $1,43081 \mathrm{E}-05$ \\
\hline Observaciones & 53 \\
\hline
\end{tabular}

\begin{tabular}{|l|c|c|c|c|c|}
\cline { 2 - 6 } \multicolumn{1}{c|}{} & \multicolumn{5}{c|}{ ANÁLISIS DE VARIANZA } \\
\cline { 2 - 6 } \multicolumn{1}{c|}{} & Grados de libertad & Suma de cuadrados & Promedio de los cuadrados & F & Valor crítico de $\mathbf{F}$ \\
\hline Regresión & 1 & $2,07595 \mathrm{E}-10$ & $2,07595 \mathrm{E}-10$ & 1,014031323 & 0,318692968 \\
\hline Residuos & 51 & $1,04408 \mathrm{E}-08$ & $2,04722 \mathrm{E}-10$ & & \\
\hline Total & 52 & $1,06484 \mathrm{E}-08$ & & & \\
\hline
\end{tabular}

\begin{tabular}{|l|c|c|c|c|c|c|c|c|}
\cline { 2 - 9 } \multicolumn{1}{c|}{} & Coeficientes & Error típico & Estadístico t & Probabilidad & Inferior 95\% & Superior 95\% & Inferior 95,0\% & Superior 95,0\% \\
\hline Intercepción & $7,57266 \mathrm{E}-05$ & $1,61655 \mathrm{E}-05$ & 4,684460749 & $2,11972 \mathrm{E}-05$ & $4,3273 \mathrm{E}-05$ & 0,00010818 & $4,3273 \mathrm{E}-05$ & 0,00010818 \\
\hline Beta 2006 & 0,158644516 & 0,157543097 & 1,006991223 & 0,318692968 & $-0,157636449$ & 0,474925481 & $-0,157636449$ & 0,474925481 \\
\hline
\end{tabular}

Estimación Beta año 2007

\begin{tabular}{|l|c|}
\hline \multicolumn{2}{|c|}{ Estadísticas de la regresión } \\
\hline Coeficiente de correlación múltiple & 0,33957008 \\
\hline Coeficiente de determinación $\mathrm{R}^{\wedge} 2$ & 0,115307839 \\
\hline $\mathrm{R}^{\wedge} 2$ ajustado & 0,095201199 \\
\hline Error típico & $3,95262 \mathrm{E}-06$ \\
\hline Observaciones & 46 \\
\hline
\end{tabular}

\begin{tabular}{|l|c|c|c|c|c|}
\cline { 2 - 6 } \multicolumn{1}{c|}{} & \multicolumn{5}{c|}{ ANÁLISIS DE VARIANZA } \\
\cline { 2 - 6 } \multicolumn{1}{c|}{} & Grados de libertad & Suma de cuadrados & Promedio de los cuadrados & F & Valor crítico de F \\
\hline Regresión & 1 & $8,95964 \mathrm{E}-11$ & $8,95964 \mathrm{E}-11$ & 5,734813912 & 0,020956541 \\
\hline Residuos & 44 & $6,87423 \mathrm{E}-10$ & $1,56232 \mathrm{E}-11$ & & \\
\hline Total & 45 & $7,77019 \mathrm{E}-10$ & & & \\
\hline
\end{tabular}

\begin{tabular}{|l|c|c|c|c|c|c|c|c|}
\cline { 2 - 8 } \multicolumn{1}{c|}{} & Coeficientes & Error típico & Estadístico t & Probabilidad & Inferior 95\% & $\begin{array}{c}\text { Superior } \\
\mathbf{9 5 \%}\end{array}$ & $\begin{array}{c}\text { Inferior } \\
\mathbf{9 5 , 0} \%\end{array}$ & $\begin{array}{c}\text { Superior } \\
\mathbf{9 5 , 0} \%\end{array}$ \\
\hline Intercepción & $1,26061 \mathrm{E}-05$ & $2,24175 \mathrm{E}-05$ & 0,562334148 & 0,576742245 & $-3,25733 \mathrm{E}-05$ & $5,77855 \mathrm{E}-05$ & $-3,25733 \mathrm{E}-05$ & $5,77855 \mathrm{E}-05$ \\
\hline Beta 2007 & 0,572570508 & 0,239094348 & 2,39474715 & 0,020956541 & 0,090707512 & 1,054433503 & 0,090707512 & 1,054433503 \\
\hline
\end{tabular}

27 Fuente: Elaboración de los autores a partir de datos de la investigación 
Estimación Beta año 2008

\begin{tabular}{|l|r|}
\hline \multicolumn{2}{|c|}{ Estadísticas de la regresión } \\
\hline Coeficiente de correlación múltiple & 0,889844954 \\
\hline Coeficiente de determinación $\mathrm{R}^{\wedge} 2$ & 0,791824042 \\
\hline $\mathrm{R}^{\wedge} 2$ ajustado & 0,787660523 \\
\hline Error típico & $8,85489 \mathrm{E}-06$ \\
\hline Observaciones & 52 \\
\hline
\end{tabular}

\begin{tabular}{|l|c|c|c|c|c|}
\cline { 2 - 6 } \multicolumn{1}{c|}{} & \multicolumn{5}{c|}{ ANÁLISIS DE VARIANZA } \\
\cline { 2 - 6 } \multicolumn{1}{c|}{} & Grados de libertad & Suma de cuadrados & Promedio de los cuadrados & F & Valor crítico de F \\
\hline Regresión & 1 & $1,4912 \mathrm{E}-08$ & $1,4912 \mathrm{E}-08$ & 190,1814331 & $1,147 \mathrm{E}-18$ \\
\hline Residuos & 50 & $3,92046 \mathrm{E}-09$ & $7,84091 \mathrm{E}-11$ & & \\
\hline Total & 51 & $1,88324 \mathrm{E}-08$ & & & \\
\hline
\end{tabular}

\begin{tabular}{|l|c|c|c|c|c|c|c|c|}
\cline { 2 - 8 } \multicolumn{1}{c|}{} & Coeficientes & Error típico & Estadístico t & Probabilidad & Inferior 95\% & Superior 95\% & $\begin{array}{c}\text { Inferior } \\
\mathbf{9 5 , 0 \%}\end{array}$ & $\begin{array}{c}\text { Superior } \\
\mathbf{9 5 , 0} \%\end{array}$ \\
\hline Intercepción & $-5,29238 \mathrm{E}-05$ & $1,02574 \mathrm{E}-05$ & $-5,159583449$ & $4,27406 \mathrm{E}-06$ & $-7,35264 \mathrm{E}-05$ & $-3,23213 \mathrm{E}-05$ & $-7,35264 \mathrm{E}-05$ & $-3,23213 \mathrm{E}-05$ \\
\hline Beta 2008 & 1,228833437 & 0,089106413 & 13,79062845 & $1,147 \mathrm{E}-18$ & 1,049857939 & 1,407808935 & 1,049857939 & 1,407808935 \\
\hline
\end{tabular}

Estimación Beta año 2009

\begin{tabular}{|l|r|}
\hline \multicolumn{2}{|c|}{ Estadísticas de la regresión } \\
\hline Coeficiente de correlación múltiple & 0,980118053 \\
\hline Coeficiente de determinación $R^{\wedge} 2$ & 0,960631398 \\
\hline$R^{\wedge} 2$ ajustado & 0,959844026 \\
\hline Error típico & $3,62291 \mathrm{E}-06$ \\
\hline Observaciones & 52 \\
\hline
\end{tabular}

\begin{tabular}{|l|c|c|c|c|c|}
\cline { 2 - 6 } \multicolumn{1}{c|}{} & \multicolumn{5}{c|}{ ANÁLISIS DE VARIANZA } \\
\cline { 2 - 6 } \multicolumn{1}{c|}{} & Grados de libertad & Suma de cuadrados & Promedio de los cuadrados & F & Valor crítico de F \\
\hline Regresión & 1 & $1,60137 \mathrm{E}-08$ & $1,60137 \mathrm{E}-08$ & 1220,047648 & $8,65792 \mathrm{E}-37$ \\
\hline Residuos & 50 & $6,56273 \mathrm{E}-10$ & $1,31255 \mathrm{E}-11$ & & \\
\hline Total & 51 & $1,667 \mathrm{E}-08$ & & & \\
\hline
\end{tabular}

\begin{tabular}{|l|c|c|c|c|c|c|c|c|}
\cline { 2 - 8 } \multicolumn{1}{c|}{} & Coeficientes & Error típico & Estadístico t & Probabilidad & Inferior 95\% & Superior 95\% & Inferior 95,0\% & Superior 95,0\% \\
\hline Intercepción & $-4,09551 \mathrm{E}-05$ & $3,39945 \mathrm{E}-06$ & $-12,04756025$ & $2,12381 \mathrm{E}-16$ & $-4,77831 \mathrm{E}-05$ & $-3,41271 \mathrm{E}-05$ & $-4,77831 \mathrm{E}-05$ & $-3,41271 \mathrm{E}-05$ \\
\hline Beta 2009 & 1,110752116 & 0,03180012 & 34,92918047 & $8,65792 \mathrm{E}-37$ & 1,046879696 & 1,174624535 & 1,046879696 & 1,174624535 \\
\hline
\end{tabular}


Estimación Beta año 2010

\begin{tabular}{|l|r|}
\hline \multicolumn{2}{|c|}{ Estadísticas de la regresión } \\
\hline Coeficiente de correlación múltiple & 0,903045434 \\
\hline Coeficiente de determinación $R^{\wedge} 2$ & 0,815491055 \\
\hline$R^{\wedge} 2$ ajustado & 0,811800876 \\
\hline Error típico & $2,72792 \mathrm{E}-06$ \\
\hline Observaciones & 52 \\
\hline
\end{tabular}

\begin{tabular}{|c|c|c|c|c|c|}
\hline & \multicolumn{5}{|c|}{ ANÁLISIS DE VARIANZA } \\
\hline & Grados de libertad & Suma de cuadrados & Promedio de los cuadrados & $\mathbf{F}$ & Valor crítico de $\mathrm{F}$ \\
\hline Regresión & 1 & $1,6445 \mathrm{E}-09$ & $1,6445 E-09$ & 220,989572 & $5,53528 \mathrm{E}-20$ \\
\hline Residuos & 50 & $3,72077 \mathrm{E}-10$ & $7,44154 \mathrm{E}-12$ & & \\
\hline Total & 51 & $2,01658 \mathrm{E}-09$ & & & \\
\hline
\end{tabular}

\begin{tabular}{|l|c|c|c|c|c|c|c|c|}
\cline { 2 - 8 } \multicolumn{1}{c|}{} & Coeficientes & Error típico & Estadístico t & Probabilidad & Inferior 95\% & Superior 95\% & Inferior 95,0\% & $\begin{array}{c}\text { Superior } \\
\mathbf{9 5 , 0 \%}\end{array}$ \\
\hline Intercepción & $-1,36632 \mathrm{E}-06$ & $3,55196 \mathrm{E}-06$ & $-0,384666838$ & 0,702115605 & $-8,50064 \mathrm{E}-06$ & $5,768 \mathrm{E}-06$ & $-8,50064 \mathrm{E}-06$ & $5,768 \mathrm{E}-06$ \\
\hline Beta 2010 & 0,688681034 & 0,046326793 & 14,86571801 & $5,53528 \mathrm{E}-20$ & 0,595630933 & 0,781731136 & 0,595630933 & 0,781731136 \\
\hline
\end{tabular}

